\title{
The ESCRT machinery regulates the secretion and long-range activity of Hedgehog
}

Tamás Matusek ${ }^{1,2,3 *}$, Franz Wendler ${ }^{1,2,3 *}$, Sophie Polès ${ }^{1,2,3}$, Sandrine Pizette ${ }^{1,2,3}$, Gisela D’Angelo ${ }^{1,2,3}$, Maximilian Fürthauer ${ }^{1,2,3}$ \& Pascal P. Thérond ${ }^{1,2,3}$

The conserved family of Hedgehog $(\mathrm{Hh})$ proteins acts as short- and long-range secreted morphogens, controlling tissue patterning and differentiation during embryonic development ${ }^{1}$. Mature Hh carries hydrophobic palmitic acid and cholesterol modifications essential for its extracellular spreading ${ }^{2}$. Various extracellular transportation mechanisms for Hh have been suggested, but the pathways actually used for Hh secretion and transport in vivo remain unclear. Here we show that $\mathrm{Hh}$ secretion in Drosophila wing imaginal discs is dependent on the endosomal sorting complex required for transport (ESCRT) ${ }^{3}$. In vivo the reduction of ESCRT activity in cells producing Hh leads to a retention of $\mathrm{Hh}$ at the external cell surface. Furthermore, we show that ESCRT activity in $\mathrm{Hh}$-producing cells is required for long-range signalling. We also provide evidence that pools of Hh and ESCRT proteins are secreted together into the extracellular space in vivo and can subsequently be detected together at the surface of receiving cells. These findings uncover a new function for ESCRT proteins in controlling morphogen activity and reveal a new mechanism for the transport of secreted Hh across the tissue by extracellular vesicles, which is necessary for long-range target induction.

Diffusible signals, such as secreted morphogens, are key players in intercellular communication during development. The Hh morphogen has a critical role in patterning the anterior-posterior axis of the developing epithelial wing imaginal discs (WIDs) in Drosophila, by specifying the presumptive intervein domain between vein 3 and vein $4(\mathrm{v} 3-\mathrm{v} 4)$ through both short- and long-range activity ${ }^{1}$. Mechanisms proposed to control spreading of $\mathrm{Hh}$ include the formation of multimers, loading on filopodia, and packaging into lipoprotein particles ${ }^{4-7}$. However, the possibility of $\mathrm{Hh}$ transport via membranous vesicles released by cells (exovesicles) has never been functionally explored. Exovesicles may be generated by plasma membrane budding or by fusion of multivesicular bodies (MVBs) with the plasma membrane, leading to the release of MVB intraluminal vesicles into the extracellular space $e^{8-10}$. Both plasma membrane budding and the formation of MVB intraluminal vesicles require the severing of thin membrane stalks by a highly conserved specialized machinery, the ESCRT complex ${ }^{3}$.

In WIDs, Hh is produced and secreted by the epithelial cells of the posterior compartment. It induces a concentration-dependent graded response in anterior cells, through activation of short-range target genes, such as the transcription factor engrailed (en) and the Hh receptor patched $(p t c)$, and long-range target genes, such as decapentaplegic $(d p p)$, the Drosophila homologue of TGF- $\beta^{11}$ and iroquois (iro $)^{12}$. The strong cell death and tumorigenic phenotype induced by the lack of ESCRT function in WIDs ${ }^{13-16}$ made it impossible for us to analyse its role in Hh secretion with classical loss-of-function mutants. We therefore used a temporally controllable RNA interference (RNAi) method restricted to Hhproducing cells and developed a 'tester line' (Methods) in which increasing Hh production in the posterior cells of the WID induced the ectopic production of $d p p$ in the most anterior cells of the WID, leading to severe outgrowth of the anterior compartment (Fig. 1a and Extended Data Fig. 1a-d). Moreover, we also observed an enlargement of the endogenous $d p p$ expression domain at the anterior-posterior border (10-12 cells wide instead of 6-8 cells wide in the wild type; Fig. 1b, c), leading to an increase in the size of the v3-v4 intervein space of the adult wings (Extended
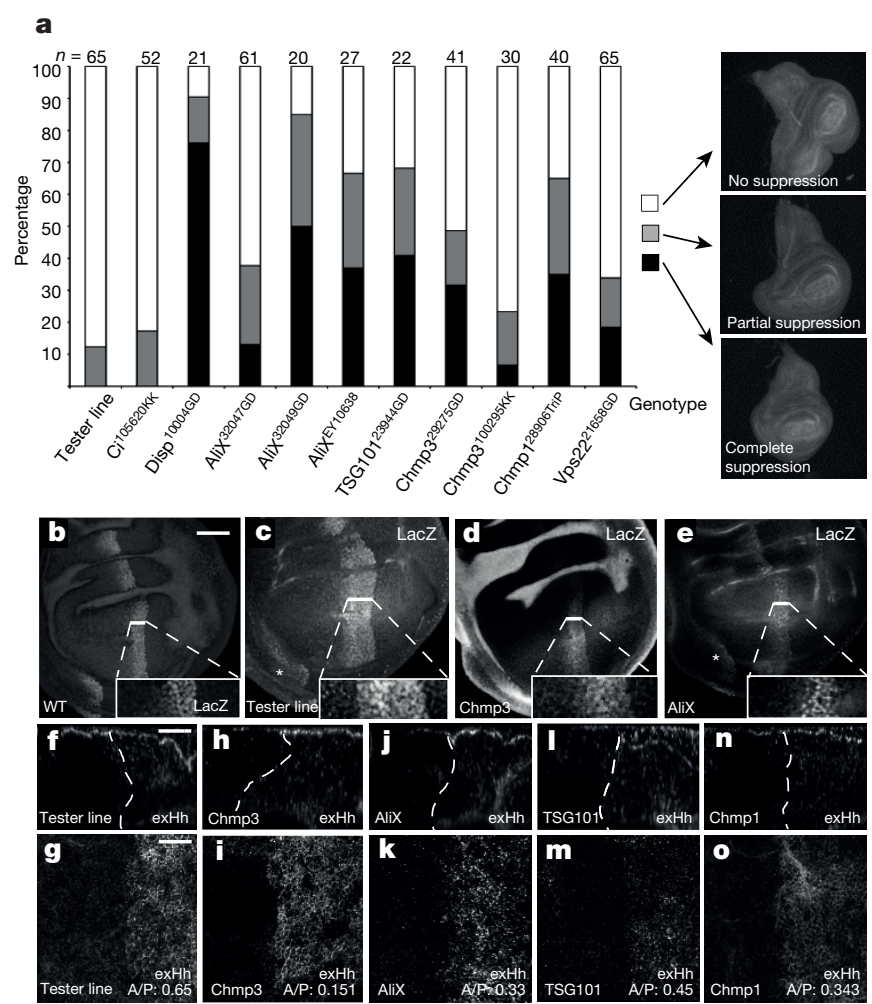

(

Figure 1 ESCRT proteins are required for the long-range activity of $\mathrm{Hh}$. a, Percentage of outgrown, moderately and fully rescued WIDs with respect to their genotypes. The number of dissected discs $(n)$ is shown at the top of the columns. Representative photographs of each category are shown on the right. Negative control: RNAi against Cubitus interruptus (Ci), which is not expressed in the posterior compartment. Positive control: RNAi against Disp. Superscripts refer to the transformant identity of the RNAi line. $\mathbf{b}-\mathbf{e}, d p p-l a c Z$ expression in wild-type (WT), tester line and tester line plus ESCRT RNAi discs. A magnification of the $d p p$-lacZ stripe is shown for each genotype in the bottom-right corner. Note that not only the cental $d p p$ stripe but also the ectopic $d p p$ expression is suppressed in tester line plus ESCRT RNAi discs (white asterisks on $\mathbf{c}$ and $\mathbf{e}$ ). Owing to the low level of Dpp staining, signal intensity was increased in d. Scale bar, $50 \mu \mathrm{m}$. $\mathbf{f}-\mathbf{o}$, Representative $X-Z$

$(\mathbf{f}, \mathbf{h}, \mathbf{j}, \mathbf{l}, \mathbf{n})$ and $X-Y-Z(\mathbf{g}, \mathbf{i}, \mathbf{k}, \mathbf{m}, \mathbf{o})$ sections showing extracellular Hh staining in control discs and discs with tester line plus ESCRT RNAi. On $X-Z$ sections (scale bar, $20 \mu \mathrm{m}$ ), apical is towards the top. The anterior-posterior border is labelled with a white dashed line. On $X-Y-Z$ sections (scale bar, $15 \mu \mathrm{m}$ ), the anterior-posterior $(\mathrm{A} / \mathrm{P})$ ratio of $\mathrm{Hh}$ staining intensity is indicated in the bottom-right corner for all genotypes. In all images anterior is to the left and in $\mathbf{b}-\mathbf{e}$ dorsal is to the top. Information about optical sections shown in all figures can be found in Supplementary Table 3. ex, extracellular. 
Data Fig. 1e-h). These two phenotypes were rescued by RNAi targeting the only known regulator of Hh secretion, Dispatched ${ }^{17}$ (Disp) (Fig. 1a and Extended Data Fig. 1e, i).

Expression in Hh-producing cells of RNAi against ESCRT proteins, including AliX, TSG101, Vps22 (also known as Larsen), Chmp1 and Chmp3 (members of the ESCRT complexes I, II and III; see Supplementary Table 1) abolished the Hh-dependent $\mathrm{v} 3-\mathrm{v} 4$ intervein space enlargement and WID anterior outgrowth (up to $48 \%$ of the WID showed complete rescue) (Fig. 1a and Extended Data Fig. 1e, j-k) without changing the level of Hh protein (Extended Data Fig. 1m, n). Rescued discs with wild-type appearance did not display any sign of apoptosis or cell-architecture defects, with the exception of Vps22, investigation of which was not further pursued (Extended Data Fig. 2a-k). Distribution of DE-cadherin (also known as Shotgun), Disp, and the glypican Dally-like (Dlp) in cells depleted for ESCRT function were also not affected (Extended Data Fig. 2h-s), indicating that the ESCRT depletion conditions used did not induce pleiotropic or indirect effects on Hh secretion.

The rescue of WID morphology revealed a role of the ESCRT proteins on the regulation of expression of the Hh long-range target $d p p$. Indeed, very anterior ectopic expression of $d p p$ and the broadening of its central stripe at the anterior-posterior border were abolished (Fig. 1d, e). Surprisingly, the expression of short-range targets requiring high-level signalling, such as En and Ptc, was still extended over up to six cells (Extended Data Fig. 3a-1). Consistent with a role of ESCRT in the longrange activity of $\mathrm{Hh}$, accumulations of $\mathrm{Hh}$ at the external apical side of Hh-producing cells were observed upon depletion of ESCRT in posterior cells whereas the extracellular apical Hh levels in the anterior receiving cells were decreased compared with wild-type discs (Fig. 1f-o).

The requirement of ESCRT function for Hh long-range activity prompted us to test whether ESCRT function was also required in animals in which $\mathrm{Hh}$ is expressed at physiological levels. We thus depleted Chmp1, AliX, TSG101 and Vps32 (also known as Shrub) by RNAi (Supplementary Table 1) in the posterior cells of an otherwise wild-type disc. We observed a significant decrease of $d p p$ and iro expression, whereas the domains of Ptc and En expression were similar to those in the wild type (Fig. 2a-e, v, x and Extended Data Fig. 4a-j, k). We obtained similar results by using the temperature-sensitive allele of the $h h$ gene $\left(h h^{t s 2}\right)$, which increases the sensitivity for defects in Hh secretion (Extended Data Figs 11, 4l-t).
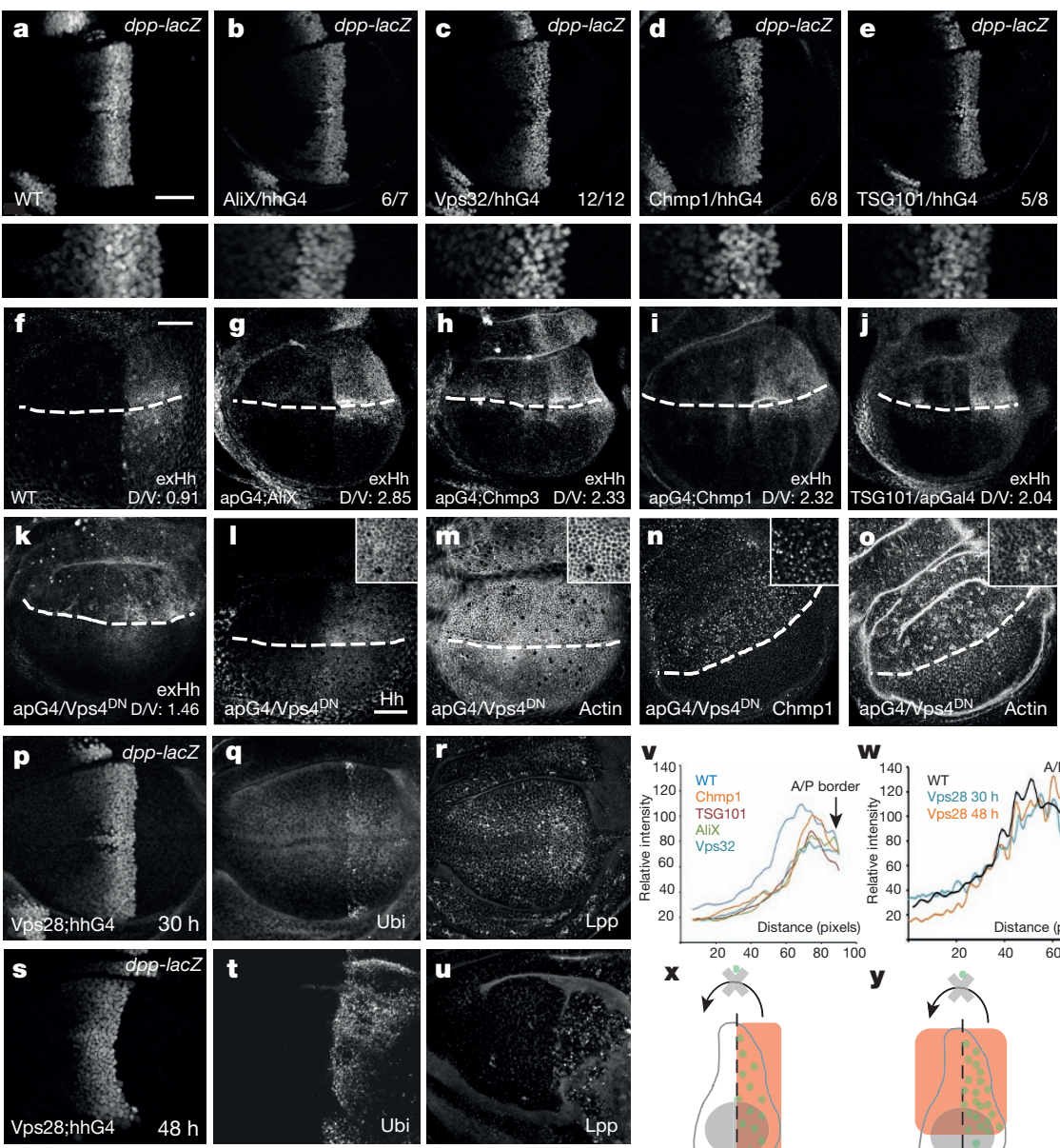
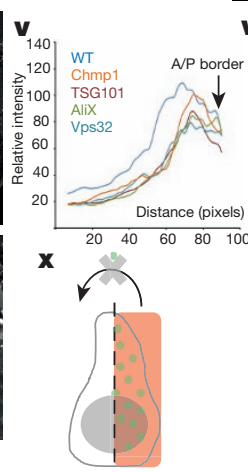

Figure $2 \mid$ Lack of ESCRT function in posterior cells leads to defects in Hh secretion and target gene induction. a-e, Pattern of $d p p$-lac $Z$ expression in WIDs with $30 \mathrm{~h}$ of RNAi against the indicated ESCRT components. A magnification of the $d p p$-lac $Z$ stripe is shown in the bottom panels. b-e, Numbers at bottom right indicate number of discs displaying reduced $d p p$ activity per whole population analysed. $\mathbf{f}-\mathbf{j}$, Extracellular staining of endogenous Hh in wild-type (WT) discs and in discs with the indicated ESCRT RNAi. Dorsal is to the top and anterior to the left. $\mathbf{f}-\mathbf{k}$, The dorsal-ventral (D/V) ratio of $\mathrm{Hh}$ staining intensity is indicated in the bottom-right corner for all genotypes. $\mathbf{k}-\mathbf{m}$, Extracellular (exHh; $\mathbf{k})$ and conventional $(\mathbf{l}-\mathbf{m})$ Hh staining in discs expressing $\mathrm{Vps} 4^{\mathrm{DN}}$. n, o, Chmp1 accumulates in endosomal compartments. F-actin staining is used to outline the cell cortex (m, o). Magnified views of dorsal-posterior cells are shown in the top-right corner. f-o, The dorsal-ventral boundary is marked with a white dashed line. $\mathbf{p}-\mathbf{u}$, Misexpression of Vps28 RNAi for $30 \mathrm{~h}(\mathbf{p}-\mathbf{r})$ or $48 \mathrm{~h}(\mathbf{s}-\mathbf{u})$ in Hh-producing cells. $\mathbf{v}$, Quantification of $d p p$-lac $Z$ from a-e. A/P, anterior-posterior. w, Quantification of $d p p$-lacZ expression in Vps28 RNAi discs presented in $\mathbf{p}, \mathbf{s} . \mathbf{x}, \mathbf{y}$, Schemes presenting the expression domain (red) of the compartment-specific Gal4 driver lines hedgehog-Gal4 (hhGal4; x) and apterous-Gal4 (apGal4; y). Green circles indicate Hh protein. Scale bars, $30 \mu \mathrm{m}$. 
To test whether impaired Hh activity was associated with defects of Hh subcellular localization, we depleted ESCRT proteins in the dorsal compartment of the WID (Fig. 2y). This led to the accumulation of Hh in dorsal ESCRT-depleted cells compared with their ventral wild-type neighbours. Accumulating $\mathrm{Hh}$ can be detected using a non-permeabilizing antibody staining protocol (see Methods), suggesting that the molecule is retained at the outer plasma membrane surface of producing cells (Fig. 2f-j). To confirm this, we induced the expression of a dominantnegative form of the ESCRT III protein Vps $4\left(\mathrm{Vps}^{\mathrm{DN}}\right)$ to block the disassembly and recycling of the ESCRT complex ${ }^{18-20}$ in Hh-secreting cells. Surprisingly, intracellular Hh was not trapped in maturation-deficient endosomes labelled with Chmp1, but instead accumulated at the apical cortex of Hh-producing cells (Fig. 2k-o). This suggests that the ESCRTdependent regulation of $\mathrm{Hh}$ secretion might not be related to the function of ESCRT proteins in MVB biogenesis. To confirm this, we analysed the effect of depletion of ESCRT function on MVB biogenesis. Blocking MVB function by expression of ESCRT RNAi or of Vps $4^{\mathrm{DN}}$ in Hhproducing cells led to the accumulation of poly-ubiquitinated epitopes (poly-Ubi) on proteins trafficking through degradative $\mathrm{MVBs}^{21}$ (Extended Data Fig. 5a-e, n-p). Interestingly, upon expression of Vps28 RNAi, this enrichment was not associated with a decrease in $d p p$ target gene expression (Fig. 2p-q, s-t, w). We also showed that an increase in poly-Ubi accumulation in Vps32-depleted discs does not correlate with a decrease in $d p p$-lacZ expression (Extended Data Fig. 5m-p). Conversely, in AliXand Vps32-depleted discs showing no accumulation of ubiquitinated proteins, expression of the long-range target $d p p$ was reduced (Fig. $2 \mathrm{~b}$, c, v and Extended Data Fig. 5j, m). This indicates that defects in MVB trafficking do not correlate with defects in Hh signalling, but suggests that a subset of ESCRT proteins regulate Hh secretion in a manner that is independent of MVB biogenesis.

Hh can be associated with the Lipophorin (Lpp) lipid carrier (which labels lipoprotein particles) secreted from the fat body ${ }^{5}$; therefore, we wondered whether trafficking of Lpp is affected in the absence of ESCRTs. We found that depletion of Vps28-which has no effect on Hh signalling (Fig. 2p, s, w) -affects Lpp trafficking (Fig. 2r, u), whereas depletion of AliX and Vps32-which affects Hh secretion and signalling (Fig. 2b, c, g) - did not perturb Lpp trafficking (Extended Data Fig. 5k, l). In accordance with the fact that ESCRT-dependent Hh secretion is independent of Lpp, only the knockdown of ESCRT proteins but not the knockdown of Lpp led to the accumulation of $\mathrm{Hh}$ at the apical pole of the cells (Fig. 2g-l and Extended Data Fig. 5q-t). These data suggest that perturbation of the ESCRT machinery impairs Hh trafficking and secretion independently of Lpp.

To investigate whether secretion of $\mathrm{Hh}$ relies on the ESCRT-dependent biogenesis of exovesicle carriers, we used a cell line ( $\mathrm{Cl} 8$ cells) derived from the WID and modified to express $\mathrm{Hh}$ in an inducible manner ${ }^{22}$. Biochemical fractionation by differential centrifugation of the $\mathrm{Hh}$-containing conditioned medium was carried out (Methods and Fig. 3a). Western blot analysis indicated that the bulk of the Hh protein present in the conditioned medium was split equally between a 120,000g supernatant (S120) and a detergent-soluble pellet (P120) (Fig. 3a). Higher-speed centrifugation for a longer time gave similar fractionation (Extended Data Fig. 6a). The S120 fraction corresponds to a soluble Hh complex with a molecular weight of $160-200 \mathrm{kDa}$ (ref. 22). The analysis by transmission electron microscopy of the $\mathrm{P} 120$ fraction from $\mathrm{Hh}$-expressing $\mathrm{Cl} 8$ cells or of $\mathrm{P} 120$ from $\mathrm{Cl} 8$ cells not expressing $\mathrm{Hh}$ showed cup-shaped vesicles of 40 $200 \mathrm{~nm}$ in diameter (Fig. 3b). Incubation of Hh-responsive Cl8 naive cells with an equivalent relative amount of P120 Hh or S120 Hh stimulated phosphorylation of the Fused protein - which reflects Hh signalling activation ${ }^{1}$ - to similar extents (Fig. $3 \mathrm{c}$ and Extended Data Fig. 6a, b), suggesting that these two fractions correspond to two different pools of Hh with similar capacities to induce Hh signalling. P120 Hh was also confined to a $35 \%(\mathrm{w} / \mathrm{v})$ Optiprep step in a density gradient, corresponding to an intermediate density (between 1.184 and $1.195 \mathrm{~g} \mathrm{ml}^{-1}$ ), indicating specific membrane association (Fig. 3d). Anti-Hh staining by immunoelectron microscopy on non-permeabilized purified P120 membranes
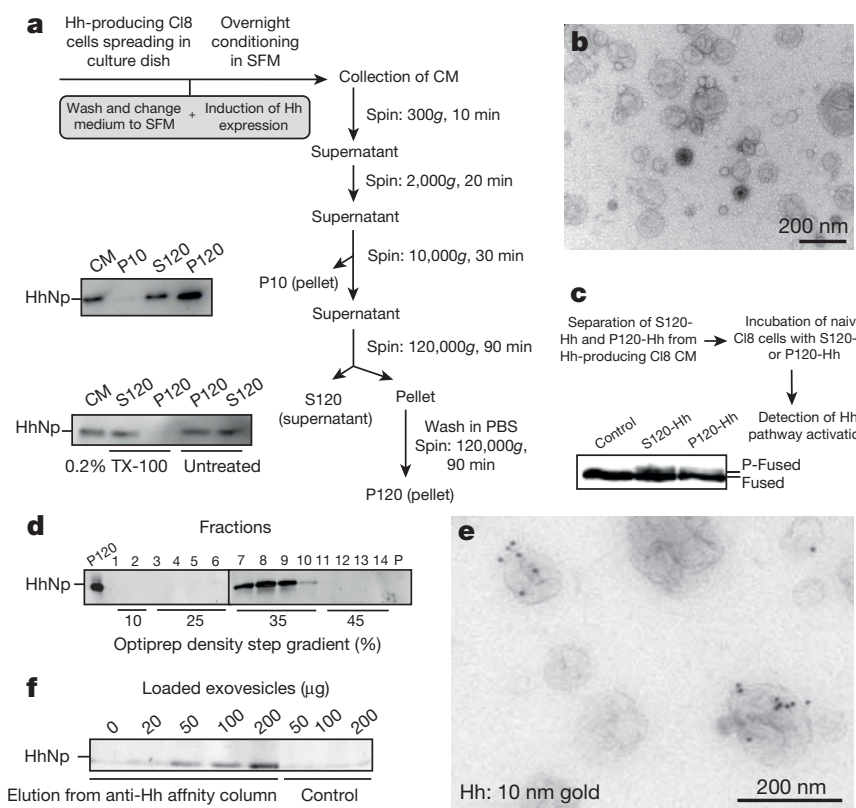

C

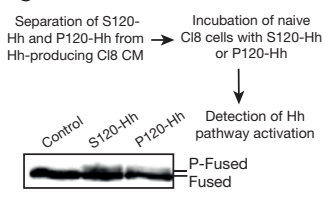

e
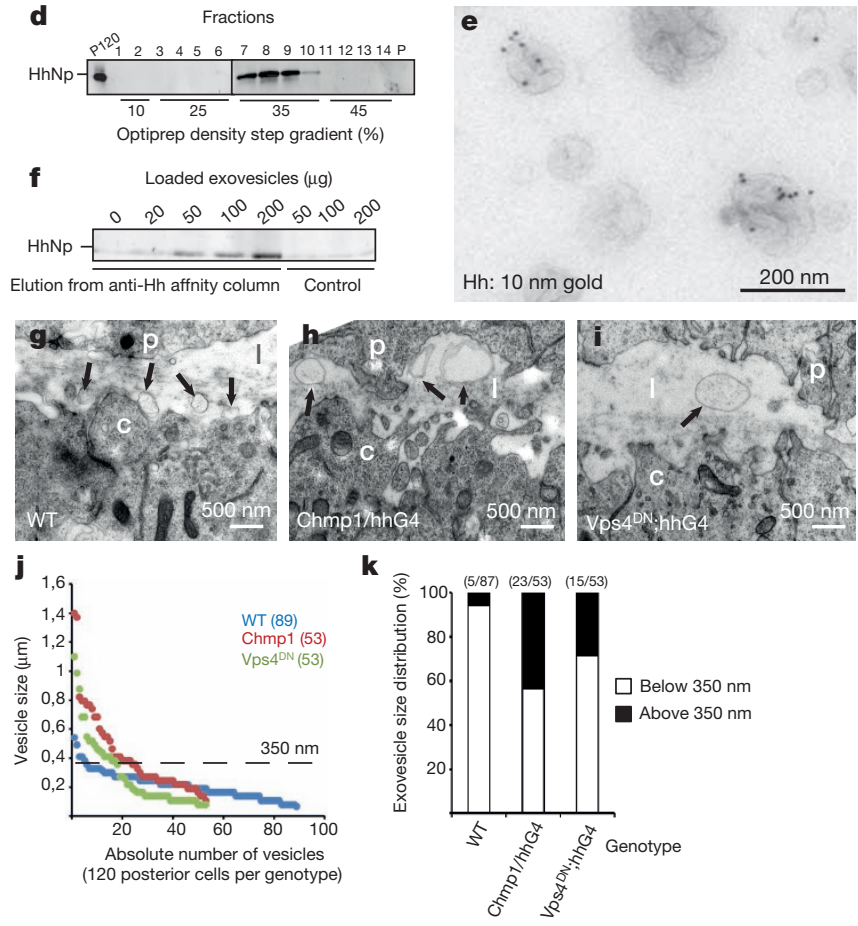

Figure $3 \mid \mathrm{Hh}$ is secreted on extracellular vesicles. a, Conditioned medium (CM) from Hh-producing $\mathrm{Cl} 8$ cells subjected to differential centrifugation. The $10,000 \mathrm{~g}$ pellet $(\mathrm{P} 10), 120,000 \mathrm{~g}$ pellet (P120) and 120,000 g supernatant (S120) fractions were analysed by immunoblotting with anti-Hh antibodies (middle). Bottom, the Hh-producing Cl8-cell S120 and P120 fractions were treated with $0.2 \%$ Triton X-100 (TX-100). HhNp, mature cholesterol-modified $\mathrm{HhN}$ peptide; SFM, serum-free medium. b, Representative transmission electron micrograph of the Hh-producing Cl8-cell P120 fraction contrast stained with $3 \%$ uranyl acetate. c, Naive $\mathrm{Cl} 8$ cells were treated with Hh-producing Cl8-cell S120 or P120 fractions or with conditioned medium from Cl8 cells (control). Cell lysates from treated cells were analysed by immunoblotting with anti-Fused antibodies. P-Fused, phosphorylated Fused. d, Hh-producing Cl8-cell P120 was fractionated on an Optiprep density step gradient from $45 \%$ to $10 \%$. e, Immunogold labelling of Hh P120 purified fraction with anti-Hh antibodies. f, Anti-Hh or control antibodies bound to Dynabeads were incubated with increasing amounts of Hh-producing Cl8-cell exovesicles from P120. Bound vesicles were eluted and analysed by immunoblotting with anti-Hh antibodies. $\mathbf{g}-\mathbf{i}$, Transmission electron micrographs of WIDs expressing Chmp1 RNAi and Vps $4^{\mathrm{DN}}$ in Hh-producing cells. White letters mark the apical side of peripodial (p) and columnar (c) cells facing the lumen (l). Black arrows indicate the luminal vesicular profiles. Scale bar, $500 \mathrm{~nm} . \mathbf{j}, \mathbf{k}$, Quantification (j) and distribution $(\mathbf{k})$ based on the size of vesicular profiles in Chmp1 RNAi and Vps $4^{\mathrm{DN}}$ compared with wild type (WT). Numbers in brackets indicate the total number of vesicular profiles counted ( $\mathbf{j}$ ), or the number of vesicular profiles sized above $350 \mathrm{~nm}$ per whole population $(\mathbf{k})$. 
revealed that $\mathrm{Hh}$ was associated with the surface of vesicles (Fig. 3e) but not on vesicles purified from non-Hh-expressing cells (Extended Data Fig. 6c). Moreover, when $\mathrm{Hh}$-containing vesicles were loaded onto a $\mathrm{Hh}$ immunoaffinity column we found that $\mathrm{Hh}$ was detectable in fractions eluted from the column, confirming the presence of Hh epitopes on the surface of vesicles (Fig. 3f).

We next identified the proteins present in the Hh-containing vesicles by performing mass spectrometry on the $\mathrm{P} 120 \mathrm{Hh}$ fraction. We identified about 160 proteins, including the $\mathrm{Hh}$ amino-terminal peptide, which contains all the signalling activity of $\mathrm{Hh}$ (Supplementary Table 2). These proteins included nine ESCRT protein members, five of them (AliX, Chmp1, Chmp3, Vps32 and Vps4) being the ones that affect Hh secretion in vivo.

We further investigated ESCRT function using electron microscopy. Careful examination of the plasma membrane and luminal space between the columnar and peripodial cells revealed the presence of extracellular vesicular profiles in the luminal space, while MVB morphology was not significantly affected (Fig. $3 \mathrm{~g}-\mathrm{k}$ and data not shown). Interestingly, these structures are bigger in size and their number is decreased in Chmp1 RNAi- and in Vps $4^{\mathrm{DN}}$-expressing discs (Fig. 3g-k). The accumulation of $\mathrm{Hh}$ at the surface of producing cells (Fig. $2 \mathrm{~g}-\mathrm{l}$ ), combined with the results of our electron microscopy study, suggest that, as for exovesicle release from HEK293T cells ${ }^{8}$ and in Caenorhabditis elegans ${ }^{9}$, Hh may be released through plasma membrane budding into the extracellular space. We therefore analysed the in vivo association of extracellular Hh with ESCRT proteins in more detail. In WIDs expressing green fluorescent protein (GFP)-tagged Vps32 in Hh-producing cells we observed dynamic Vps32-GFP-positive particles in the luminal space between columnar epithelial and squamous peripodial cells, at a distance from producing cells (Fig. 4a, b and Extended Data Fig. 7a, b). Interestingly, extracellular Hh partly colocalized with Vps32-GFP, at the apical external side of both posterior producing and anterior receiving Hh cells (Fig. 4c-e and Extended Data Fig. 8a-i). No such colocalization was observed between external $\mathrm{Hh}$ and an inner plasma membrane marker (GAP43-GFP) (Extended Data Fig. 8j-1). Moreover, endogenous Chmp1-positive external particles were observed at various places in the luminal space (Fig. 4f-h and Extended Data Figs 7c-f, 9). Importantly, extracellular Hh-GFP was found to be colocalized with endogenous Chmp1 in the luminal space (Fig. $4 \mathrm{f}-\mathrm{h}$ and Extended Data Fig. $7 \mathrm{c}-\mathrm{f})$.

It has been previously shown that $\mathrm{Hh}$ is present in the fly haemolymph ${ }^{23}$ which we therefore used as another in vivo model to analyse Hh secretion. In this tissue, we found similar overlap between Vps32-GFP and endogeneous Hh on secreted particles (Fig. 4i-k). Such overlap was also found with endogeneous AliX (for validation of the anti-AliX antibody see Extended Data Fig. 5i), but not with GAP43-GFP or Vps36-GFP (Extended Data Fig. 10), suggesting that, in this tissue also, Hh is associated with particles that are positive for a subset of ESCRT proteins.

If Hh is secreted together with ESCRT proteins, then misexpression of the Hh-receptor Ptc should trap both Hh and ESCRT proteins at the cell surface. We investigated this possibility by expressing a mutant form of Ptc, Ptc(1130X), in the Hh-receiving cells at the anterior-posterior border. Ptc(1130X) can bind and sequester external Hh but cannot be internalized. Consistent with a potential role of Chmp1-positive exovesicles in the delivery of $\mathrm{Hh}$ to its target tissue, $\mathrm{Ptc}(1130 \mathrm{X})$ expression led to the trapping of endogenous Chmp1 and Hh at the surface of recipient cells in the anterior compartment (Fig. 4l-n). By contrast, Ptc(1130X) misexpression did not cause any detectable trapping of Lpp (Fig. 4o-q), indicating that ESCRT/Hh-positive exovesicles are distinct from previously described Lpp-positive Hh carriers ${ }^{5}$.

Our findings provide evidence for a previously unidentified mechanism for Hh release and intercellular communication (see Supplementary Discussion). Overall, our results suggest a new mechanism for morphogen secretion, in which a pool of signalling molecules follows an ESCRTdependent route in morphogen-producing cells, contributing to the establishment of a morphogen gradient. We believe that this mechanism
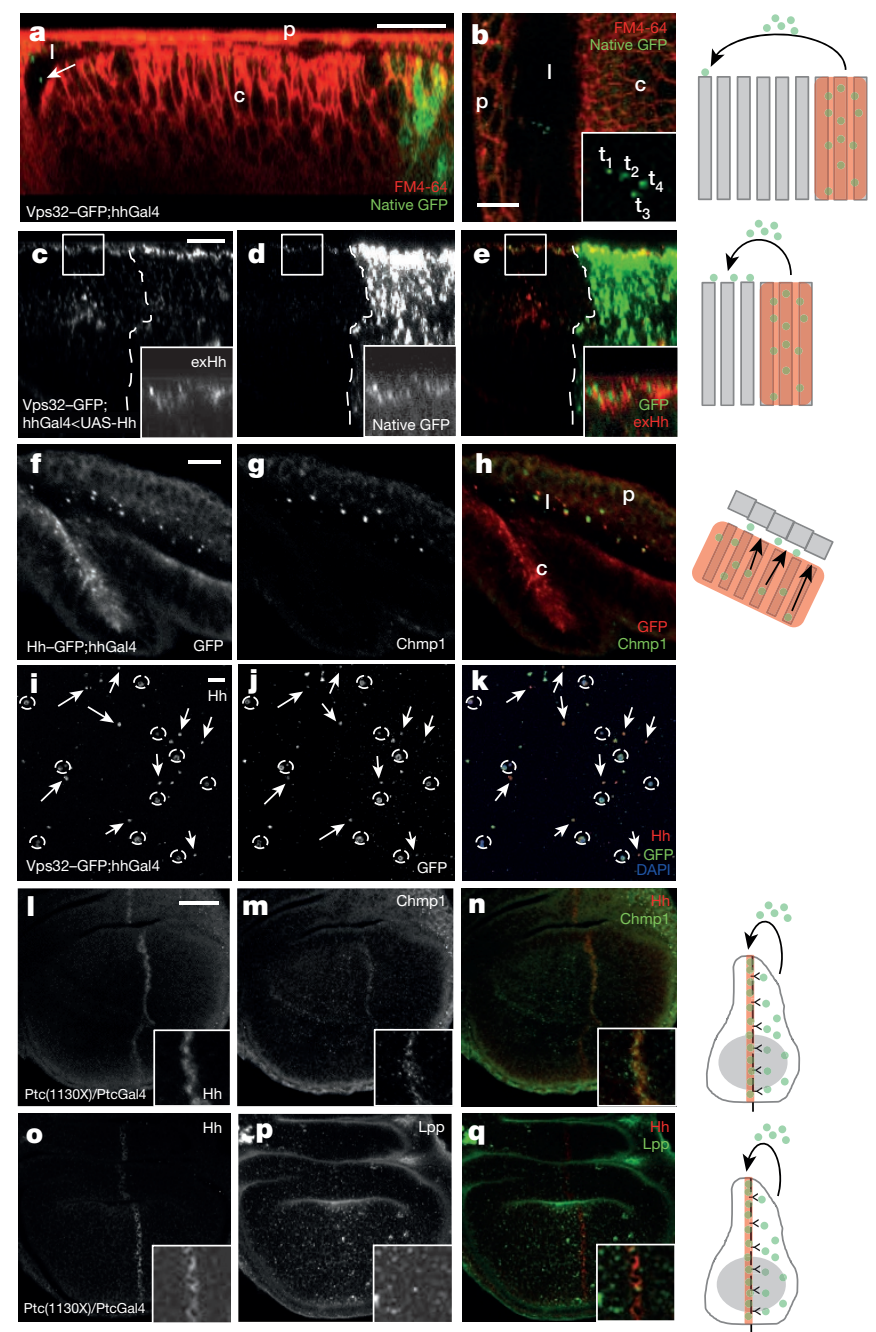

Figure $4 \mid$ ESCRT-positive exovesicles transport $\mathrm{Hh}$ in the extracellular space of Drosophila wing discs and haemolymph. a, $X-Z$ section of a living WID in which Vps32-GFP is expressed in the Hh-secreting posterior compartment (green cells on the right). The arrow indicates a Vps32-positive particle in the lumen. Scale bar, $20 \mu \mathrm{m}$. Plasma membrane was labelled with the vital membrane dye FM4-64 [( $N$-(3-triethylammoniumpropyl)-4-(6-(4(diethylamino) phenyl) hexatrienyl) pyridinium dibromide)]. b. Temporal projection of a time-lapse video of a Vps32-GFP particle moving through the luminal space. Single time points (acquisition time: $0.77 \mathrm{~s}$ per frame) are indicated in the bottom right corner (t1-t4). Scale bar, $10 \mu \mathrm{m}$. c-e, $X-Z$ section of a disc showing partial colocalization of extracellular $\mathrm{Hh}$ (exHh and Vps32-GFP in Hh-receiving cells. Apical is to the top, anterior to the left. The anterior-posterior border is labelled with a white dashed line. Scale bar, $10 \mu \mathrm{m}$. Pearson coefficient: 0.576; M1M2 coefficients: 0.308 (Hh) and 0.593 (Vps32-GFP). $\mathbf{f}-\mathbf{h}$, Colocalization of Hh-GFP and endogenous Chmp1 protein in particles in the luminal space (see also Extended Data Fig. 7). Scale bar, $10 \mu \mathrm{m}$. i-k, Colocalization of $\mathrm{Hh}$ and Vps32-GFP in non-cellular particles within the haemolymph. White circles mark 4',6-diamidino-2phenylindole (DAPI)-positive blood cells, white arrows indicate examples of colocalization. Scale bar, $20 \mu \mathrm{m}$. I-q, Trapping of Hh and Chmp1 but not Lpp at Ptc(1130X)-expressing cells. Scale bar, $20 \mu \mathrm{m}$. c, columnar cells; l, lumen; p, peripodial cells. Magnification is shown in the bottom-right corner of $\mathbf{c}-\mathbf{e}$, 1-n and o-q. Schemes on the right column indicate position of columnar cells (in red) that express the drivers used in a-q. Green circles indicate $\mathrm{Hh}$, and black branches indicate Ptc(1130X) proteins.

of Hh secretion may be conserved, as the vertebrate counterpart of $\mathrm{Hh}$, Sonic hedgehog (Shh), has been observed on exovesicles in the mouse ventral node ${ }^{24}$. However, it remains to be determined whether mammalian ESCRTs are involved in this process and whether functionally active vesicular Shh spreads through the extracellular space. 
Online Content Methods, along with any additional Extended Data display items and Source Data, are available in the online version of the paper; references unique to these sections appear only in the online paper.

\section{Received 27 September 2013; accepted 8 September 2014.}

1. Briscoe,J. \& Thérond, P.P. The mechanisms of Hedgehog signalling and its roles in development and disease. Nature Rev. Mol. Cell Biol. 14, 416-429 (2013).

2. Mann, R. K. \& Beachy, P. A. Novel lipid modifications of secreted protein signals. Annu. Rev. Biochem. 73, 891-923 (2004).

3. Rusten, T. E., Vaccari, T. \& Stenmark, H. Shaping development with ESCRTs. Nature Cell Biol. 14, 38-45 (2012).

4. Zeng, X. et al. A freely diffusible form of Sonic hedgehog mediates long-range signalling. Nature 411, 716-720 (2001)

5. Panáková, D., Sprong, H., Marois, E., Thiele, C. \& Eaton, S. Lipoprotein particles are required for Hedgehog and Wingless signalling. Nature 435, 58-65 (2005).

6. Sanders, T. A., Llagostera, E. \& Barna, M. Specialized filopodia direct long-range transport of SHH during vertebrate tissue patterning. Nature 497, 628-632 (2013).

7. Bischoff, M. et al. Cytonemes are required for the establishment of a normal Hedgehog morphogen gradient in Drosophila epithelia. Nature Cell Biol. 15, 1269-1281 (2013)

8. Nabhan, J. F., Hu, R., Oh, R. S., Cohen, S. N. \& Lu, Q. Formation and release of arrestin domain-containing protein 1-mediated microvesicles (ARMMs) at plasma membrane by recruitment of TSG101 protein. Proc. Natl Acad. Sci. USA 109 4146-4151 (2012).

9. Wehman, A. M., Poggioli, C., Schweinsberg, P., Grant, B. D. \& Nance, J. The P4-ATPase TAT- 5 inhibits the budding of extracellular vesicles in C. elegans embryos. Curr. Biol. 21, 1951-1959 (2011).

10. Raposo, G. \& Stoorvogel, W. Extracellular vesicles: exosomes, microvesicles, and friends. J. Cell Biol. 200, 373-383 (2013).

11. Torroja, C., Gorfinkiel, N. \& Guerrero, I. Mechanisms of Hedgehog gradient formation and interpretation. J. Neurobiol. 64, 334-356 (2005).

12. Gómez-Skarmeta, J. L. \& Modolell, J. araucan and caupolican provide a link between compartment subdivisions and patterning of sensory organs and veins in the Drosophila wing. Genes Dev. 10, 2935-2945 (1996).

13. Vaccari, T. \& Bilder, D. The Drosophila tumor suppressor vps 25 prevents nonautonomous overproliferation by regulating notch trafficking. Dev. Cell $\mathbf{9}$, 687-698 (2005).

14. Herz, H.-M. et al. vps25 mosaics display non-autonomous cell survival and overgrowth, and autonomous apoptosis. Development 133, 1871-1880 (2006)

15. Thompson, B. J. et al. Tumor suppressor properties of the ESCRT-II complex component Vps25 in Drosophila. Dev. Cell 9, 711-720 (2005).

16. Vaccari, T. et al. Comparative analysis of ESCRT-I, ESCRT-II and ESCRT-III function in Drosophila by efficient isolation of ESCRT mutants. J. Cell Sci. 122, 2413-2423 (2009).

17. Burke, R. et al. Dispatched, a novel sterol-sensing domain protein dedicated to the release of cholesterol-modified hedgehog from signaling cells. Cell 99, 803-815 (1999).
18. Babst, M., Sato, T. K., Banta, L. M. \& Emr, S. D. Endosomal transport function in yeast requires a novel AAA-type ATPase, Vps4p. EMBO J. 16, 1820-1831 (1997).

19. Fujita, $\mathrm{H}$. et al. A dominant negative form of the AAA ATPase SKD1/VPS4 impairs membrane trafficking out of endosomal/lysosomal compartments: class $E$ vps phenotype in mammalian cells. J. Cell Sci. 116, 401-414 (2003).

20. Rusten, T. E. et al. ESCRTs and Fab1 regulate distinct steps of autophagy. Curr. Biol. 17, 1817-1825 (2007)

21. Raiborg, C. \& Stenmark, H. The ESCRT machinery in endosomal sorting of ubiquitylated membrane proteins. Nature 458, 445-452 (2009).

22. Gallet, A., Ruel, L., Staccini-Lavenant, L. \& Thérond, P. P. Cholesterol modification is necessary for controlled planar long-range activity of Hedgehog in Drosophila epithelia. Development 133, 407-418 (2006).

23. Palm, W. et al. Secretion and signaling activities of lipoprotein-associated Hedgehog and non-sterol-modified Hedgehog in flies and mammals. PLoS Biol. 11, e1001505 (2013)

24. Tanaka, Y., Okada, Y. \& Hirokawa, N. FGF-induced vesicular release of Sonic hedgehog and retinoic acid in leftward nodal flow is critical for left-right determination. Nature 435, 172-177 (2005).

Supplementary Information is available in the online version of the paper.

Acknowledgements This work was supported by the French Government (National Research Agency, ANR) through the "Investments for the Future" LABEX SIGNALIFE (program reference number ANR-11-LABX-0028-01) and by the Fondation pour la Recherche Médicale (reference number DEQ20110421324). T.M. and F.W. were supported by the Fondation Association pour la Recherche Contre le Cancer (ARC) and Ligue Nationale Contre le Cancer, respectively. M.F. was supported by ATIP/Avenir, ARC and the Human Frontier Science Program. The authors acknowledge S. Pagnotta for technical support. We thank K. Haglund for sharing the anti-AliX antibody before publication. We thank L. Staccini-Lavenant and M.-A. Derieppe for technical help.

Author Contributions P.P.T. conceived the project and supervised the study. P.P.T. and T.M. designed the genetic studies. T.M. performed all the genetic tests and most of the immunohistochemistry on WIDs. F.W. and P.P.T. designed the biochemical studies. F.W. performed the biochemistry/molecular biology experiments. M.F. designed and performed the live imaging experiments and generated the Chmp1b antibody. M.F. and S.Po. analysed the localization of Hh, Chmpl and Lpp in wild-type animals and in discs expressing Vps4 ${ }^{\mathrm{DN}}$, Ptc(1130X) and Lpp RNAi. G.D'A. produced reagents and contributed to the experimental design of extracellular labelling of Hh. S.Pi. performed the electron microscopy and immunoelectron microscopy analysis, and performed the counting on the electron microscopy data. P.P.T., M.F., F.W., S.Pi. and T.M. analysed the individual collected data sets. P.P.T. and T.M. assembled and edited the figures. P.P.T. wrote the manuscript. All authors commented on the manuscript versions. S.Pi. and S.Po. contributed equally to this work.

Author Information Reprints and permissions information is available at www.nature.com/reprints. The authors declare no competing financial interests. Readers are welcome to comment on the online version of the paper. Correspondence and requests for materials should be addressed to P.P.T. (therond@unice.fr). 


\section{METHODS}

Fly stocks and genetics. Fly stocks were grown under standard conditions. For investigations of the effect of ESCRT RNAi in Hh gain-of-function conditions, the $w ; t u b G a l 80^{t s} ; h h$ Gal4 $<U A S-H h / T M 6 b, T b$ 'tester line' was crossed with either control or ESCRT RNAi lines. Starter crosses were flipped every $12 \mathrm{~h}$ at $25^{\circ} \mathrm{C}$, to synchronize the progeny, and vials with L2 instar larvae were transferred to $29^{\circ} \mathrm{C}$. The induction of upstream activating sequence (UAS)-Hh expression in this setup leads to strong pharate adult lethality, the amount of eclosed escaper flies in the control $w ; t u b G a l 80^{t s} /+; h h$ Gal4 $<U A S-H h /+$ genotype is below $1 \%$. For the WID morphology assay, non-Tb larvae were dissected at the L3 larval instar stage. As a control for suppression of Hh-dependent outgrowth, we used the Disp ${ }^{1004 \mathrm{GD}} \mathrm{RNAi}$ line, which gives no phenotype in otherwise wild-type animals (data not shown), confirming the greater sensitivity of our experimental set-up for defects in Hh secretion. In Hh loss-of-function experiments we used the hhGal4 (enhancer trap for the $h h$ locus, weakly hypomorphic by itself) $/ h h^{t s 2}$ (conditional null allele ${ }^{25}$ ) heteroallelic combination, which is viable at permissive temperature $\left(18^{\circ} \mathrm{C}\right)$ and semi-lethal (up to $60 \%$ of the flies die as pharate adults) after incubation at restrictive temperature $\left(29^{\circ} \mathrm{C}\right)$ for 3 days. For simplicity, we refer in the text to $h h^{t s 2}$ for $h h^{t s 2} / h h$ Gal4. ESCRT RNAi; $h h^{t s 2} / S M 6 \hat{b} T M 6 b, T b$ males were crossed with $w ; d p p-n u c-l a c Z^{P 10638}$; $h h \mathrm{Gal} 4 / \mathrm{SM} 6 \hat{b} \mathrm{TM} 6 b, \mathrm{~Tb}$ virgin females at $18^{\circ} \mathrm{C}$. Crosses were kept at $18^{\circ} \mathrm{C}$ and flipped daily. Vials containing L1 larvae were shifted to $29^{\circ} \mathrm{C}$, and non- $\mathrm{Tb} \mathrm{L} 3$ larvae were dissected after 4 days of induction. For visualization of the changes in the levels of endogenous extracellular $\mathrm{Hh}$, wild-type control or ESCRT RNAilines were crossed with apGal4<dpp-nuc-lacZ ${ }^{\text {P10638}}$;MKRS/SM $6 \hat{b} T M 6 b, T b$. Crosses were kept at $25^{\circ} \mathrm{C}$, flipped every $12 \mathrm{~h}$, and non-Tb L3 larvae from the progeny were dissected for further analysis.

The RNAilines used in this study were: $\mathrm{Ci}^{105620 K K}$ (VDRC), Disp ${ }^{10004 \mathrm{GD}}$ (VDRC). Detailed description about the usage of ESCRT RNAi-s and further ESCRT properties can be found in Supplementary Table 1. The other alleles used were $w^{1118}$ as a wildtype control; $h h^{t s 2}$ (Bloomington \#1684), iro-lac $Z^{26}$, the nuclear $d p p-n u c-l a c Z^{P 10638}$ reporter (provided by L. Xinhua), UAS-Ptc ${ }^{1130 \mathrm{X}}$ (Bloomington \#44612) ${ }^{27}$.

Live imaging of Vps32-GFP in Drosophila WIDs. For visualization of the distribution of Vps32-GFP in living WIDs, animals of the UAS-Vps32-GFP/+; $h h \mathrm{Gal} 4<\mathrm{tubGal} 8 \mathrm{t}^{\mathrm{ts}} /+$ genotype were kept at room temperature until the late third larval instar stage and were then shifted to $29^{\circ} \mathrm{C}$ the evening (or for $6 \mathrm{~h}$ when looking at both $\mathrm{Hh}$ and Vps32-GFP distribution in fixed discs) before dissection.

Dissected WIDs were mounted in Drosophila Shields \& Sang M3 medium (Sigma) supplemented with FM4-64 (1:2,000 from $10 \mu \mathrm{gl}^{-1}$ stock, Invitrogen F34653) and $0.7 \%$ low melting point agarose.

Adult wing preparation. Adult flies were collected in glycerolethanol (3:1) and stored for at least $24 \mathrm{~h}$ before dissection. Adult wings were removed with forceps at their hinge and briefly rinsed in $96 \%$ ethanol before mounting in Euparal, as previously described ${ }^{28}$. Preparations were kept at $60{ }^{\circ} \mathrm{C}$ on a slide-heating plate overnight before image acquisition. Bright-field images were taken with a Leica Axioplan2 microscope (objective, $\times 5$; Plan NEOFLUAR; NA $=0.15$ ) equipped with a Leica DFC490 digital camera. For image acquisition, we used LAS v.2.5.0 R1 (build 975). Dissections and immunohistochemistry. Conventional staining was performed as described previously ${ }^{29}$. For imaging, WIDs were mounted in VECTASHIELD mounting medium (Vector Laboratories)

For extracellular Hh and GFP staining, L3 larvae were collected and cleaned in ice-cold PBS. Larvae were then dissected in ice-cold Schneider's medium (Sigma) and incubated with primary antibodies in Schneider's medium at $4{ }^{\circ} \mathrm{C}$ for $1 \mathrm{~h}$. Following this step, samples were rinsed three times with ice-cold PBS and fixed for $15 \mathrm{~min}$ in PBS plus $2 \%$ formaldehyde at $4{ }^{\circ} \mathrm{C}$. Fixed tissue then was washed with PBS (three times for 10 min each in PBS at room temperature) and blocked by incubation for $30 \mathrm{~min}$ in PBS plus $2 \%$ bovine serum albumin (BSA). Secondary antibodies in PBS plus 2\% BSA were added and the samples were incubated for $1 \mathrm{~h}$ at room temperature and then washed in PBS and mounted as described earlier.

For the visualization of exovesicles containing Hh-GFP and endogenous Chmp1 protein in WIDs, animals of the $h h G a l 4<t u b G a l 80^{t s} / U A S-H h$-GFP genotype were kept at room temperature until the late third larval instar stage and were then shifted to $29^{\circ} \mathrm{C}$ on the evening before dissection.

For analysis of the effect of $\mathrm{Vps} 4^{\mathrm{DN}}$ on the localization of $\mathrm{Hh}$ and $\mathrm{Chmpl}$, animals of the UAS-Vps4 $4^{D N} / t u b G a l 80^{t s}$; hh Gal4 $<U A S-H h /+$ and UAS-Vps4 $4^{D N} /$ tubGalso $0^{t s}$; $h h \mathrm{Gal} / \mathrm{H}+$ genotypes were kept at $18^{\circ} \mathrm{C}$ until the late third larval instar stage and were then shifted to $29^{\circ} \mathrm{C}$ for the last $8 \mathrm{~h}$ before dissection.

We induced Ptc(1130X) expression in Hh-receiving cells, by keeping animals of the $p t c G a l 4<t u b G a l s 0^{t s} / U A S-p t c^{1130 X}$ genotype at room temperature for $24 \mathrm{~h}$ after egg laying, after which they were shifted to $29^{\circ} \mathrm{C}$ until dissection.

For haemolymph immunohistochemistry, ten larvae were bled on ice for $1 \mathrm{~min}$ and $1 \mu \mathrm{l}$ of the haemolymph was dispersed on poly-L-lysine-coated 8-well tissue chamber slides (MAT-TEK). The preparation was then dried in vacuum for $5 \mathrm{~min}$ and immediately fixed in PBS plus $2 \%$ formaldehyde for $20 \mathrm{~min}$ at room temperature.
The fixed haemolymph was then washed with PBS three times for 5 min and incubated with PBS plus 5\% FCS for $1 \mathrm{~h}$. Following this step, slides were incubated with primary antibodies overnight at $4{ }^{\circ} \mathrm{C}$, and afterwards washed three times for 10 min with PBS. Secondary antibodies were diluted in PBS plus 5\% FCS and added to the slides for $1 \mathrm{~h}$. Finally, before mounting in VECTASHIELD supplemented with DAPI, slides were washed again three times for 10 min with PBS.

Mounted WID preparations and haemolymph were imaged with a Leica TCS SP5 confocal microscope (objective, $\times 40$; HCX PLAN APO; NA = 1.3) and the LAS AF 2.6.3.8173 program, a Zeiss LSM510 (objective, $\times 40$; PLAN APO; NA $=1.3$ ) or a ZEISS LSM780 (objective, $\times 63$; PLAN APO; NA $=1.4$ ). Bright-field WID images were taken with a Leica Axioplan2 microscope (objective, $\times 10$; Plan NEOFLUAR; $\mathrm{NA}=0.3$ ).

Antibodies used in this study. Primary antibodies used were as follows. Rabbit anti- $\mathrm{Hh}^{30}(1: 500$ from crude serum for conventional, $1: 100$ from purified serum for extracellular), rat anti-Hh (1:250, this study), mouse anti-GFP (1:1,000 for conventional, 1:50 for extracellular; Roche \#1814460), mouse anti-Dlg (1:50; DSHB 4F3), rat anti-DE-cadherin (1:50; DSHB DCAD2), chicken anti- $\beta$-gal (1:1,000; GeneTEX GTX77365), rat anti-Ci 2A1 (provided by B. Holmgren; 1:10), mouse anti-Ptc 5E10 (provided by P. Ingham; 1:400), rabbit anti-En (1:1,000; Santa Cruz sc-28640), rabbit anti-Caspase III (1:500;Cell Signaling \#9661), mouse-anti-Ubi (clone FK2, 1:1,000; ENZO Life Sciences, BML-PW8810-0100), guinea-pig-anti-AliX (1:2,000; provided by K. Haglund before publication), rabbit-anti-Lpp and guinea-pig-anti-Lpp (both 1:500; provided by S. Eaton).

Chmp1 protein was detected using a custom-made antibody (used at a dilution of 1:300) generated through immunization with an antigenic peptide (QDELSQR LAKLRDQV) corresponding to a carboxy-terminal epitope of zebrafish Chmplb that is largely conserved in Drosophila melanogaster (Eurogentec).

Secondary antibodies used were as follows. Goat anti-rabbit Alexa488 (1:500; Invitrogen A11034), goat anti-rabbit Alexa546 (1:500; Invitrogen A11035), goat antirabbit DyLight649 (1:500; Jackson Immunoresearch Laboratories 111-605-003), goat anti-rabbit Cy5 (1:200; Jackson Immunoresearch Laboratories 111-175-144), donkey anti-mouse Alexa488 (1:500; Invitrogen A21202), donkey anti-mouse Alexa546 (1:500; Invitrogen A10036), donkey anti-rat Alexa488 (Invitrogen A21208), goat anti-rat Alexa546 (1:500; Invitrogen A11081), goat anti-chicken Alexa488 (1:500; Invitrogen A11039) and TRITC-phalloidin (1:100; Sigma P1951-1MG) were used. Image analysis. Bright-field and confocal images were edited in Adobe Photoshop CS5 Extended (x64, v.12) or Fiji 1.48a. For intensity measurements, intensity profiles were originally determined with Fiji. Raw intensity values were then further processed in Microsoft Excel 2010. To calculate Pearson and M1M2 coefficients the Fiji JACoP plugin was used ${ }^{31}$

Hh exovesicle isolation and partial purification. Flasks of confluent cells (from 20 to $6075-\mathrm{cm}^{2}$ flasks) were washed the day before the experiment, with $10 \mathrm{ml}$ PBS. They were then incubated overnight with $4 \mathrm{ml}$ serum-free Schneider's medium supplemented with $100 \mu \mathrm{M} \mathrm{CuSO}_{4}$ for $\mathrm{Hh}$ expression induction. Conditioned medium was then subjected to centrifugation at $300 \mathrm{~g}$ for $10 \mathrm{~min}$ and the supernatant was centrifuged a second time at $2,000 \mathrm{~g}$ for $20 \mathrm{~min}$. The supernatant was then cleared by centrifugation at $10,000 \mathrm{~g}$ for $30 \mathrm{~min}$ and then at $120,000 \mathrm{~g}$ (a single centrifugation at $160,000 \mathrm{~g}$ gave similar results) for $90 \mathrm{~min}$. The $\mathrm{P} 120$ pellet was resuspended in PBS and centrifuged at $120,000 \mathrm{~g}$ for $90 \mathrm{~min}$. All steps were performed at $4{ }^{\circ} \mathrm{C}$. The resuspended pellet was then immediately frozen in 300-500 $\mu$ laliquots in liquid nitrogen, giving a typical protein concentration of $5-10 \mu \mathrm{g} \mu \mathrm{l}^{-1}$, and stored for up to 3 months at $-70^{\circ} \mathrm{C}$.

Typically, we loaded $25 \%$ of the P120 obtained from $1 \mathrm{ml}$ of conditioned medium per lane. An equal amount of the $\mathrm{S} 120$ pellet was loaded after TCA precipitation and resuspension in $1 \times$ loading buffer. A similar protocol was followed for the isolation of exovesicles from cells not producing $\mathrm{Hh}$. When indicated, vesicle preparations were subjected to equilibrium centrifugation. In such cases, the resuspended P120 was adjusted to $400 \mu \mathrm{l}$ of $45 \%$ (w/v) Optiprep (60\% iodixanol stock; Axis-Shield) and layered on top of a step gradient consisting of $400 \mu \mathrm{l} 35 \%, 400 \mu \mathrm{l} 25 \%$ and $200 \mu \mathrm{l} 10 \%$ Optiprep (w/v). After centrifugation for $16-18 \mathrm{~h}$ at $120,000 \mathrm{~g}, 100 \mu \mathrm{l}$ aliquots were collected, starting from the top of the tubes, and snap-frozen in liquid $\mathrm{N}_{2}$. Immunoblotting was performed as previously described ${ }^{32}$.

Immunoelectron microscopy. Fractions ( 7 to 9 from the Optiprep gradient) purified from Hh-expressing or non-expressing cells were fixed in $2 \%$ paraformaldehyde, deposited on Formvar carbon-coated electron microscopy grids and immunolabelled with rabbit anti-Hh TK antibody (a gift from T. Kornberg) and protein A-gold conjugates, as described previously $\mathrm{y}^{33}$. Exovesicles were observed at $80 \mathrm{kV}$ with a $120 \mathrm{kV}$ JEOL JEM-1400 electron microscope and digital acquisitions were obtained with a MORADA digital camera (Olympus SIS). Protein A-gold conjugates $(10 \mathrm{~nm})$ were purchased from the Cell Microscopy Center, Department of Cell Biology, Utrecht University.

Transmission electron microscopy. WIDs were fixed in $1.5 \%$ glutaraldehyde in $0.075 \mathrm{M}$ cacodylate buffer and embedded in Epon according to standard procedures. 
Seventy-nanometre sections were observed at $100 \mathrm{kV}$ with a $120 \mathrm{kV} \mathrm{JEOL} \mathrm{JEM-1400}$ electron microscope and digital acquisitions were obtained with a MORADA digital camera (Olympus SIS).

Both immuno- and transmission electron microscopy were performed on the Centre Commun de Microscopie Appliquée electron microscopy core facility (Université Nice Sophia Antipolis).

Immunoisolation. Dynabead (Life Technologies) magnetic beads already coupled to protein A were saturated with rabbit serum containing either anti-Hh or control IgGs. The prepared beads were then incubated with $\mathrm{Hh}$ vesicles from enriched fractions. The beads were washed according to the manufacturer's protocol and bound material was eluted with $0.1 \mathrm{mM}$ glycine $\mathrm{pH} 2$ and subjected to western blotting. $\mathrm{Hh}$ response assay. On the day before the assay, $\mathrm{Cl} 8$ cells were used to seed sixwell plates at 50\% confluence. The following day, the cells were washed in PBS and incubated with P120 or S120 fractions (from the equivalent of $3 \mathrm{ml}$ of Hh-producing Cl8-cell conditioned medium) for 1 day. Cells were then scraped off the plates, washed twice and lysed. The lysate was centrifuged at $12,000 \mathrm{~g}$ and the supernatant was analysed by western blotting for Fused mobility shift (anti-Fu $\left.{ }^{34} 1: 1,000\right)$. Equal relative amounts of P120 and S120 were analysed, to determine the amount of $\mathrm{Hh}$ before and after incubation with $\mathrm{Cl} 8$ cells, by western blotting (anti-Hh: 1:1,000; data not shown).

Western blot on rescued WIDs. One-hundred rescued discs of ESCRT RNAi/ tubGal80 ${ }^{\text {ts }}$; hh Gal4 $<U A S-H h /+$ genotype or controls (wild type, Disp ${ }^{10004 G D}$ ) were collected into ice-cold PBS, and extracted in Laemmli buffer. The lysate was analysed on 12\% SDS-PAGE and developed with anti-Hh and anti-Tubulin. Band density was measured with Fiji and raw data were analysed in Microsoft Excel.

Hh-P120 analysis by combined liquid chromatography with tandem mass spectrometry. Mass spectrometry analysis was performed on the Hh-containing P120 fraction obtained from Hh-producing Cl8-cell conditioned medium. Processed samples were sent to the European Molecular Biology Laboratory (EMBL) mass spectrometry core facility centre (http://www.embl.de/proteomics/proteomics_services) and were treated according to the facility protocol (detailed protocol can be provided upon request). In brief, peptides resulting from either in-gel or in-solution digestion with trypsin were analysed by combined liquid chromatography with tandem mass spectrometry (LC-MS/MS) on a nano-high-performance liquid chromatography (HPLC) system (Proxeon) coupled directly to either an LTQ Orbitrap Velos (Thermo Fisher Scientific) or MaXis Q-Tof (Bruker). Data were processed using either MaxQuant (v.1.0.13.13) or within Hystar (Bruker) to generate files that could be submitted to Mascot (Matrix Science) for database searching against a UniProt species specific database (D. melanogaster).

25. Ma, C., Zhou, Y., Beachy, P. A. \& Moses, K. The segment polarity gene hedgehog is required for progression of the morphogenetic furrow in the developing Drosophila eye. Cell 75, 927-938 (1993).

26. Letizia, A., Barrio, R. \& Campuzano, S. Antagonistic and cooperative actions of the EGFR and Dpp pathways on the iroquois genes regulate Drosophila mesothorax specification and patterning. Development 134, 1337-1346 (2007).

27. Johnson, R. L., Milenkovic, L. \& Scott, M. P. In vivo functions of the patched protein: requirement of the $\mathrm{C}$ terminus for target gene inactivation but not Hedgehog sequestration. Mol. Cell 6, 467-478 (2000).

28. Adler, P. N., Charlton, J. \& Vinson, C. Allelic variation at the frizzled locus of Drosophila. Dev. Genet. 8, 99-119 (1987).

29. Gallet, A., Ruel, L., Staccini-Lavenant, L. \& Thérond, P. P. Cholesterol modification is necessary for controlled planar long-range activity of Hedgehog in Drosophila epithelia. Development 133, 407-418 (2006).

30. Gallet, A., Rodriguez, R., Ruel, L. \& Therond, P. P. Cholesterol modification of Hedgehog is required for trafficking and movement, revealing an asymmetric cellular response to Hedgehog. Dev. Cell 4, 191-204 (2003).

31. Bolte, S. \& Cordelières, F.P.A guided tour into subcellular colocalization analysis in light microscopy. J. Microsc. 224, 213-232 (2006).

32. Ruel, L., Rodriguez, R., Gallet, A., Lavenant-Staccini, L. \& Thérond, P. P. Stability and association of Smoothened, Costal2 and Fused with Cubitus interruptus are regulated by Hedgehog. Nature Cell Biol. 5, 907-913 (2003).

33. Théry, C., Amigorena, S., Raposo, G. \& Clayton, A. Isolation and characterization of exosomes from cell culture supernatants and biological fluids. Curr. Protoc. Cell Biol. Chapter 3, Unit 3.22 (2006).

34. Thérond, P. P., Knight, J. D., Kornberg, T. B. \& Bishop, J. M. Phosphorylation of the fused protein kinase in response to signaling from hedgehog. Proc. Natl Acad. Sci. USA 93, 4224-4228 (1996). 

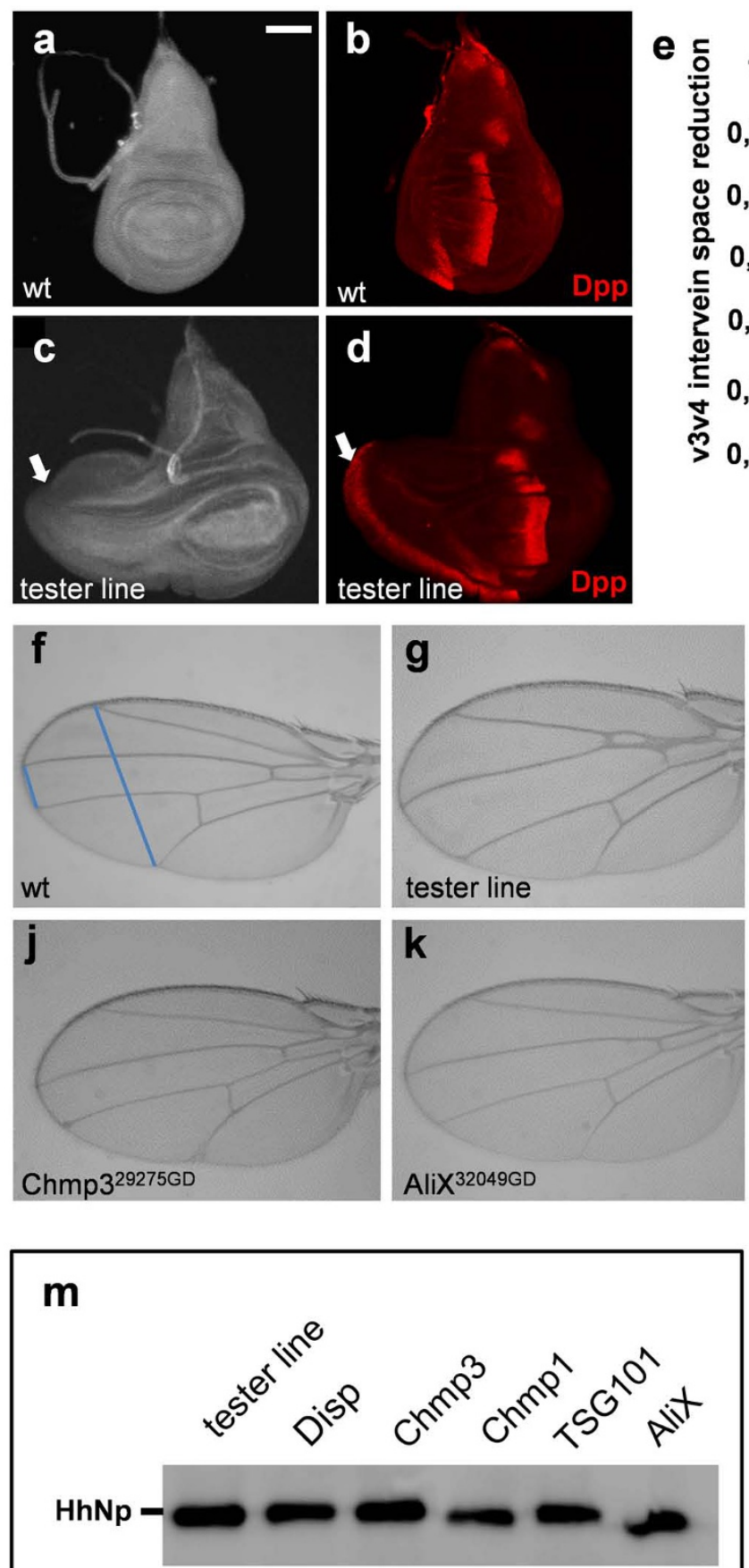

Tubulin -

Extended Data Figure $1 \mid$ ESCRT depletion in posterior cells rescues the $\mathrm{Hh}$ gain-of-function phenotype in adult Drosophila wings. a-d, Morphology and $d p p$-lacZ expression pattern of wild-type (WT; $\mathbf{a}, \mathbf{b}$ ) and Hh-

overexpressing WIDs (c, d; tester line). Scale bar, $100 \mu \mathrm{m}$. Dorsal is towards the top and anterior is to the left. The white arrow in c indicates anterior outgrowth, whereas in $\mathbf{d}$ it points to cells expressing ectopic $d p p$-lacZ. e, Quantification (mean of the ratios of absolute values) of the $\mathrm{v} 3-\mathrm{v} 4$ intervein space (shorter blue line on $\mathbf{f}$ ) relative to the whole wing span (longer blue line on $\mathbf{f}$ ) normalized to the tester line. Sample numbers (indicating the number of wings examined)
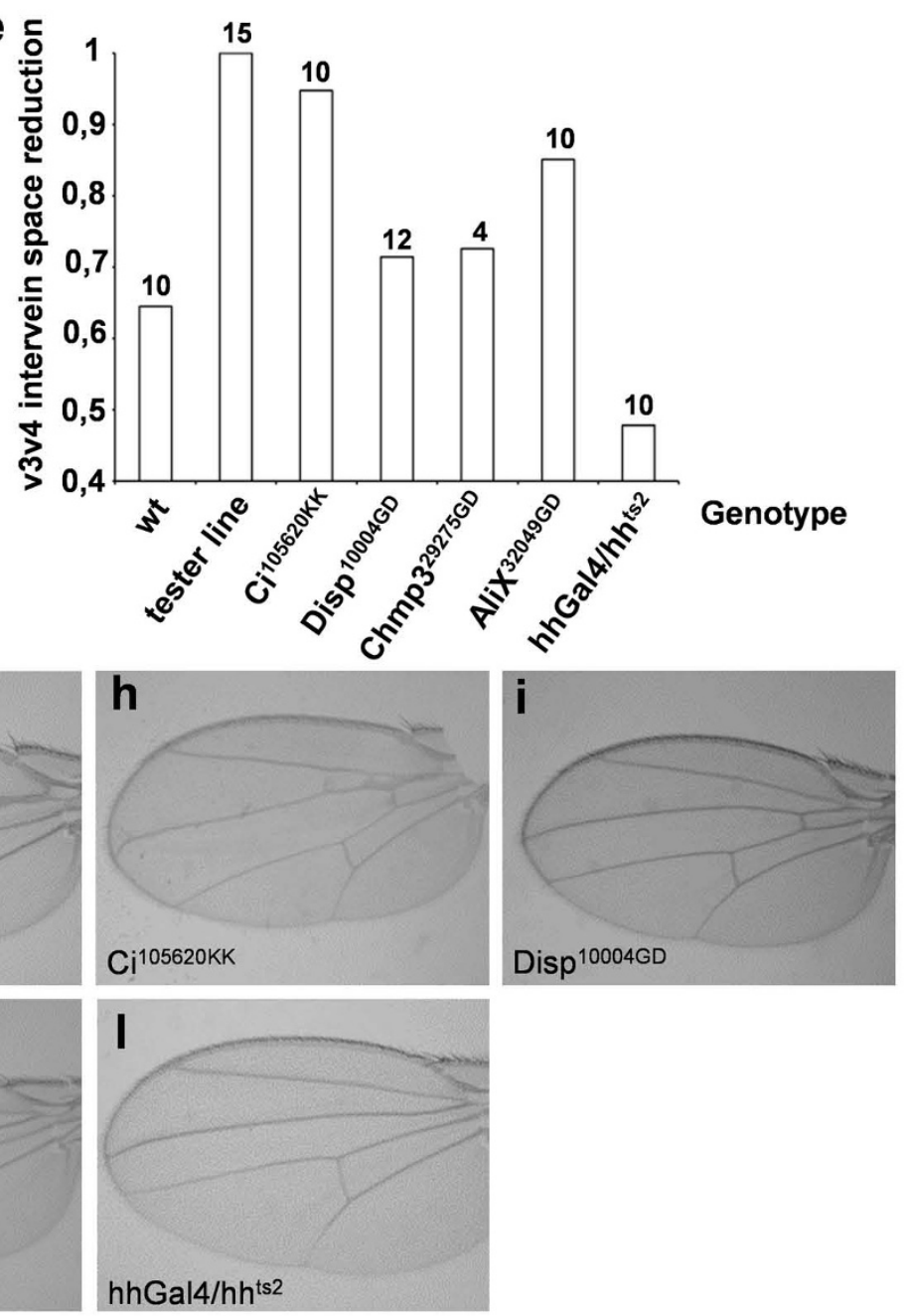

$\mathbf{n}$

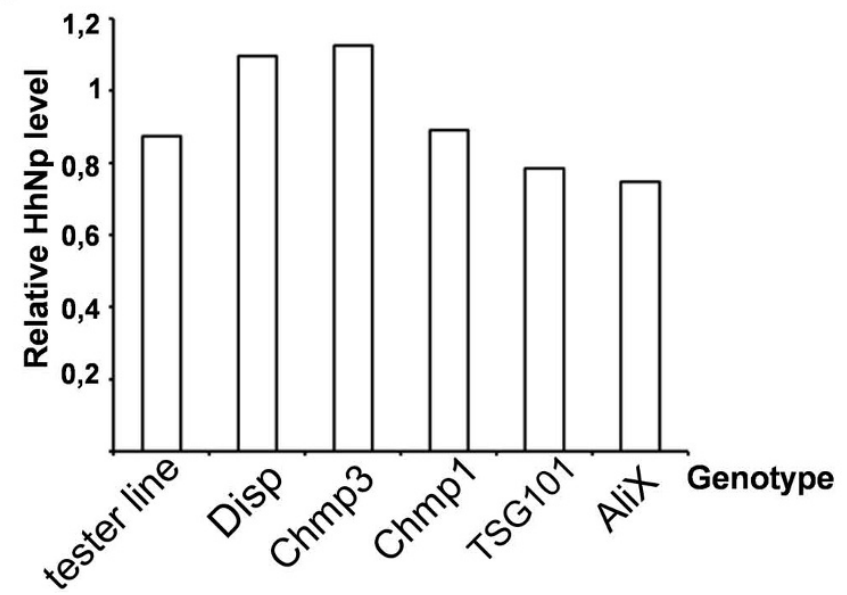

are shown at the top of each column. $\mathbf{f}-\mathbf{k}$, Representative examples of controls $(\mathbf{f}-\mathbf{i})$ and wings of tester line plus ESCRT RNAi $(\mathbf{j}, \mathbf{k})$ escapers. The amount of eclosed escaper flies in the control $w$; tubGalso ${ }^{t s} /+$; hhGal4 $<U A S$ $\mathrm{Hh} /+$ genotype is below $1 \% .1, h h$ Gal4/hh $h^{t s 2}$ mutant wing from animals kept at restrictive temperature for 4 days from L1 larval stage. $\mathbf{m}$, Representative western blot of WID lysates from rescued discs (ESCRT RNAi, UAS-Hh; hhGal4). $\mathbf{n}$, Quantification of band density from $\mathbf{m}$. HhNp values were normalized to tubulin values in each individual case. 

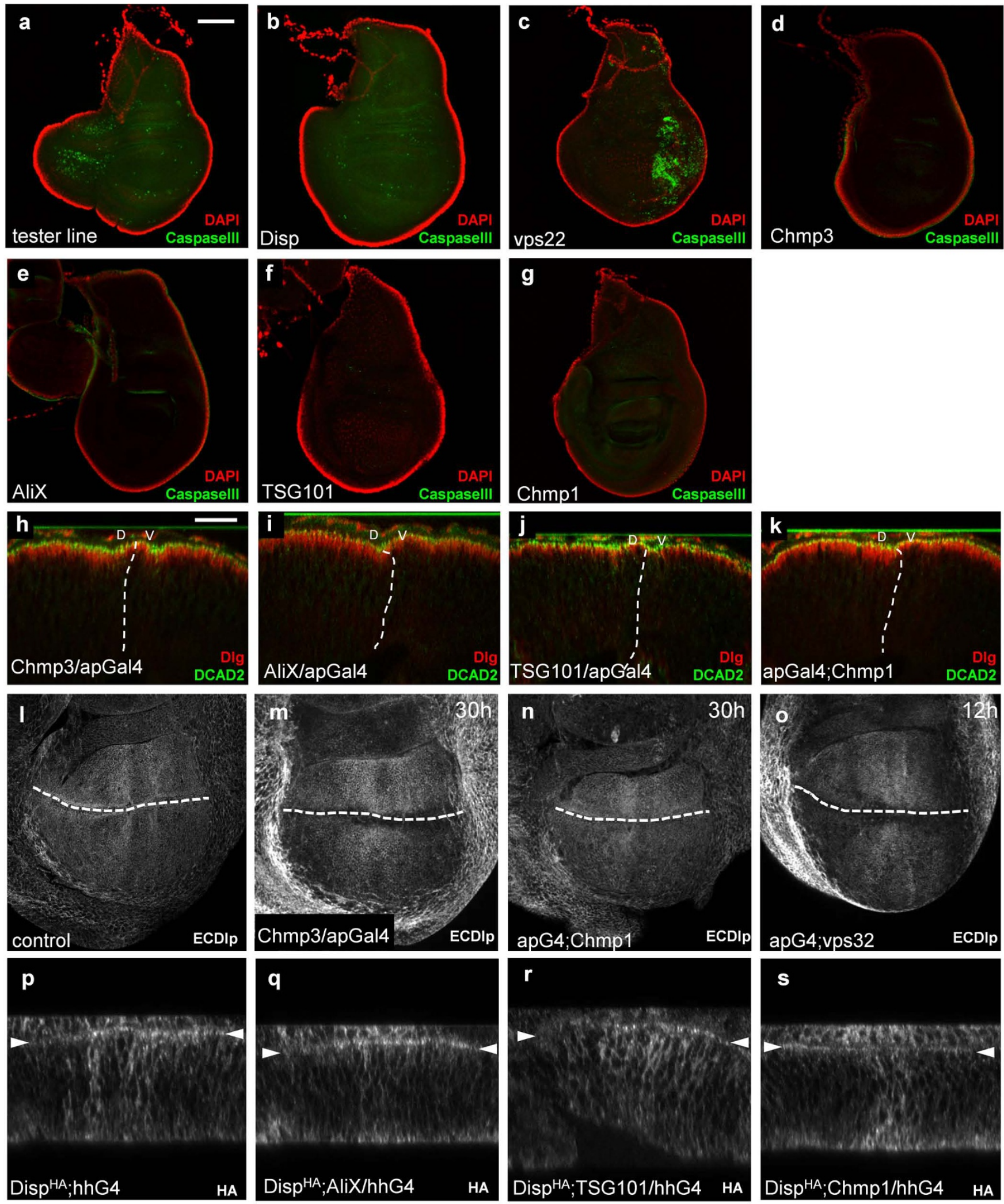

Extended Data Figure $2 \mid$ Mild ESCRT RNAi knockdown does not lead to apoptosis or cell polarity changes. $\mathbf{a}-\mathbf{g}$, Control $(\mathbf{a}, \mathbf{b})$ and ESCRT RNAi $(\mathbf{c}-\mathbf{g})$ discs stained with antibodies against the apoptotic marker Caspase III (green). Peripodial nuclei are labelled with DAPI (red). Rescued discs with wild-type appearance did not display any sign of apoptosis or cellarchitecture defects with the exception of Vps22, investigation of which was not further pursued. Scale bar, $100 \mu \mathrm{m}$. h-k, $X-Z$ section of WIDs expressing ESCRT RNAi in the dorsal compartment (D) with apGal4 driver and labelled for the polarity markers DE-cadherin (DCAD2; green) and Disc-large

(Dlg; red). The ventral domain (V) is wild type. Scale bar, $20 \mu \mathrm{m}$. l-o, Secretion of Dlp is not affected in discs expressing ESCRT RNAi in the dorsal compartment for 12-30 h. ECDlp, for extracellular Dlp protein. $\mathbf{p}-\mathbf{s}$, Disp ${ }^{\mathrm{HA}}$ subcellular localization is not affected in Hh-producing cells simultaneously expressing ESCRT RNAi for $30 \mathrm{~h}$. In all $X-Z$ images, apical is towards the top and dorsal is to the left. The dorsal-ventral boundary is indicated by a dashed white line. White arrowheads in $\mathbf{p}-\mathbf{s}$ indicate the apical side of the columnar cells. 


\section{RESEARCH LETTER}
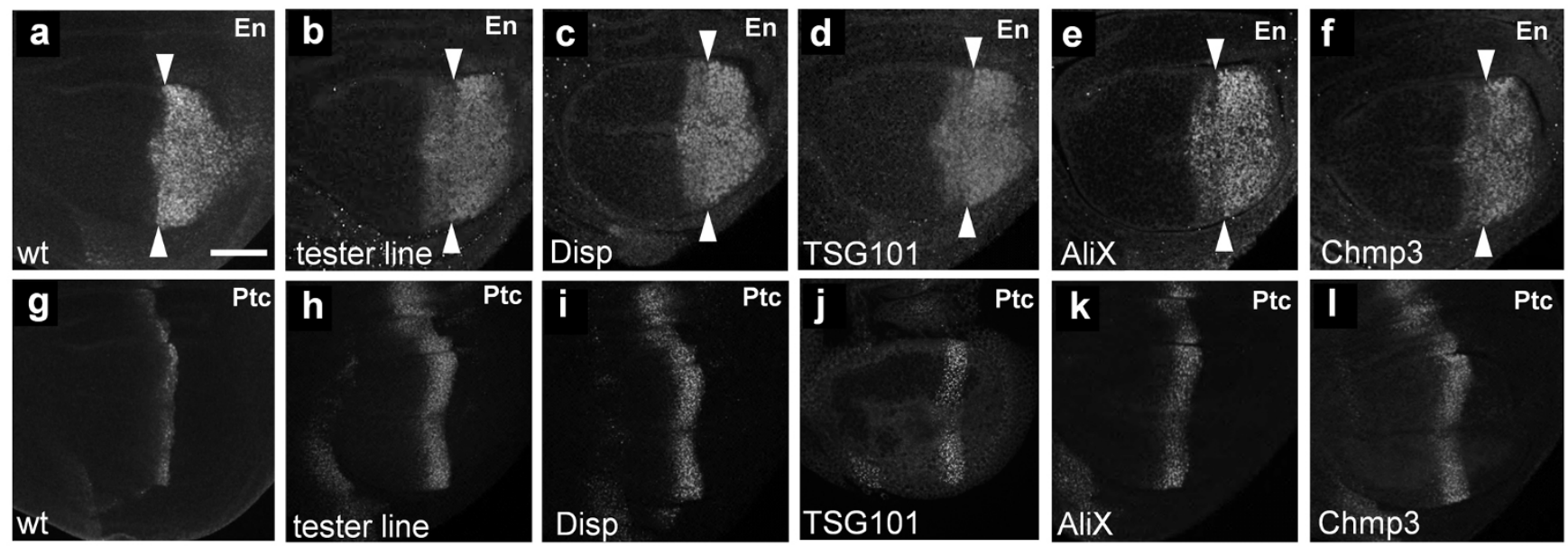

Extended Data Figure $3 \mid$ The anterior expression of Engrailed and Patched is not affected by ESCRT RNAi in conditions of Hh gain of function. a-l, In all images, dorsal is towards the top and anterior is to the left.

En $(\mathbf{a}-\mathbf{f})$ and Ptc (g-l) expression in control discs $(\mathbf{a}-\mathbf{c}, \mathbf{g}-\mathbf{i})$ and discs with tester line plus ESCRT RNAi $(\mathbf{d}-\mathbf{f}, \mathbf{j}-\mathbf{l})$ in the posterior compartment. White triangles indicate the anterior-posterior border. Scale bar, $50 \mu \mathrm{m}$. 

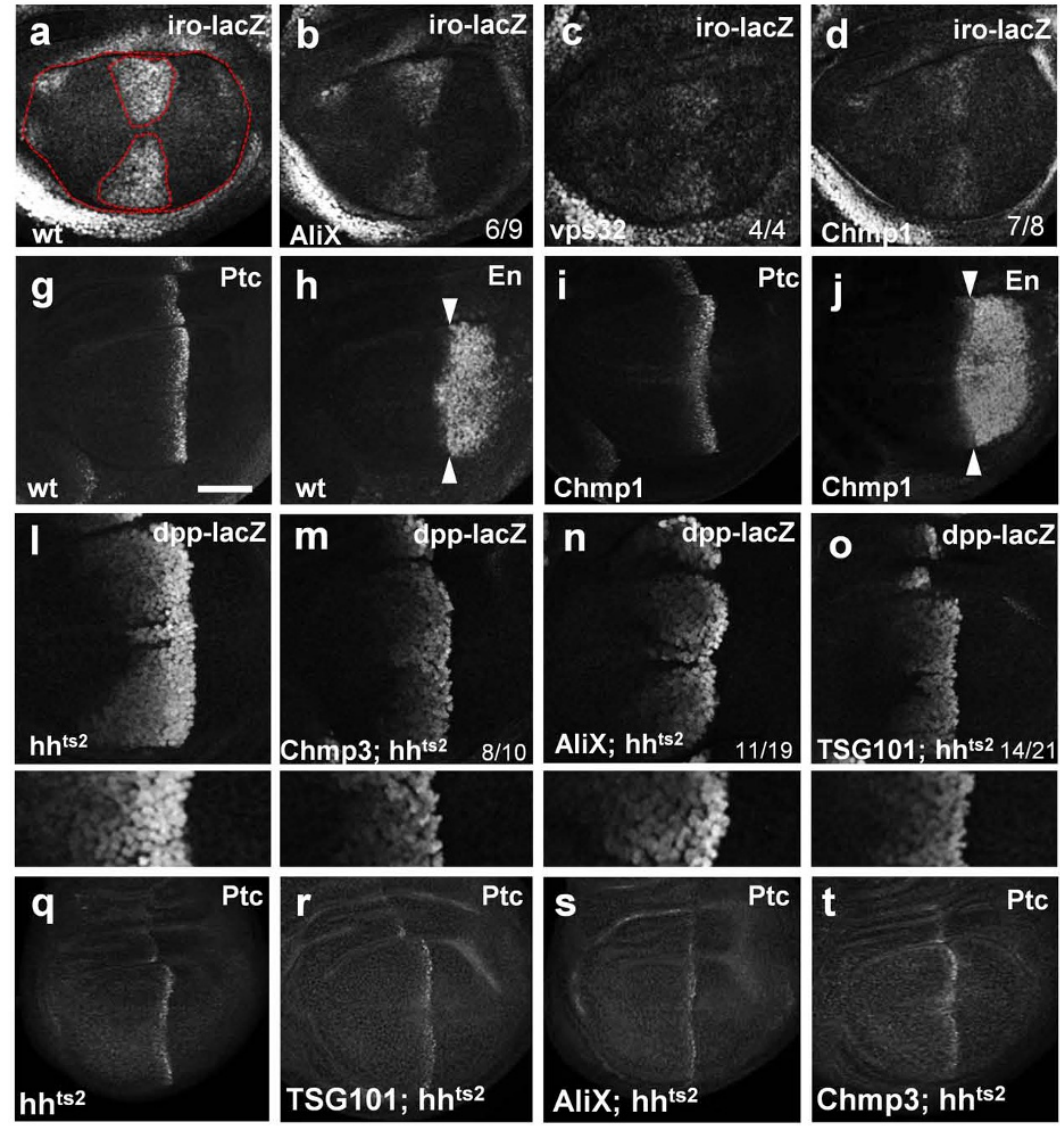

\section{(1)}
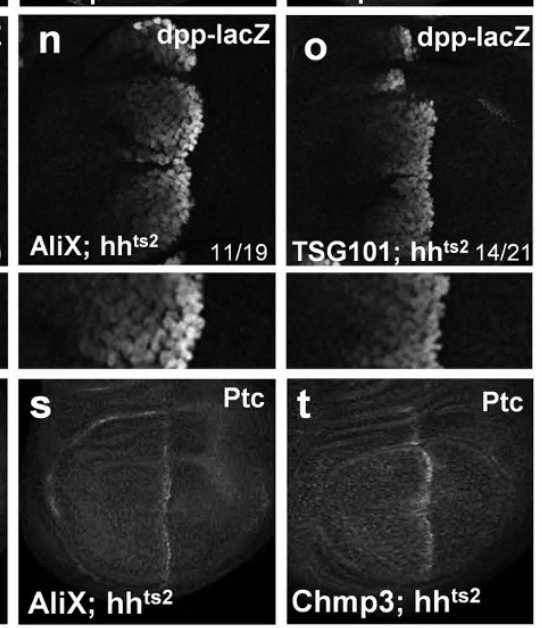

Extended Data Figure $4 \mid$ Ptc production is maintained in conditions in which iro-lac $Z$ and $d p p-l a c Z$ expression is reduced by ESCRT RNAi. a-d, The long-range target iro-lacZ expression is reduced in discs expressing ESCRT RNAi in the posterior compartment. The red dashed lines indicate the areas used for the quantification in $\mathbf{e}, \mathbf{f}$. e, $\mathbf{f}$, Quantification of the expression area (ratio of the area of iro-expressing cells to the whole WID pouch) (e) and intensity (where absolute average intensity values of iro-lacZ-expressing cells in the WID pouch were normalized to the background) (f) of iro-lacZ expression in ESCRT RNAi discs compared with wild type. $\mathbf{g}-\mathbf{j}$, Ptc (g, i) and $E n(\mathbf{h}, \mathbf{j})$ expression in wild type and conditions of Chmp1 RNAi in posterior cells. White triangles indicate the anterior-posterior $(\mathrm{A} / \mathrm{P})$ border. $\mathbf{k}$, Quantification of Ptc protein expression in discs expressing ESCRT RNAi in the posterior compartment. l-o, Representative examples of the decrease in $d p p$-lacZ expression in $h h^{t s 2}$ discs with RNAi against ESCRT in posterior cells (ESCRT RNAi; $h h^{t s 2}$ ) grown at restrictive temperature for 4 days from $\mathrm{L} 1$
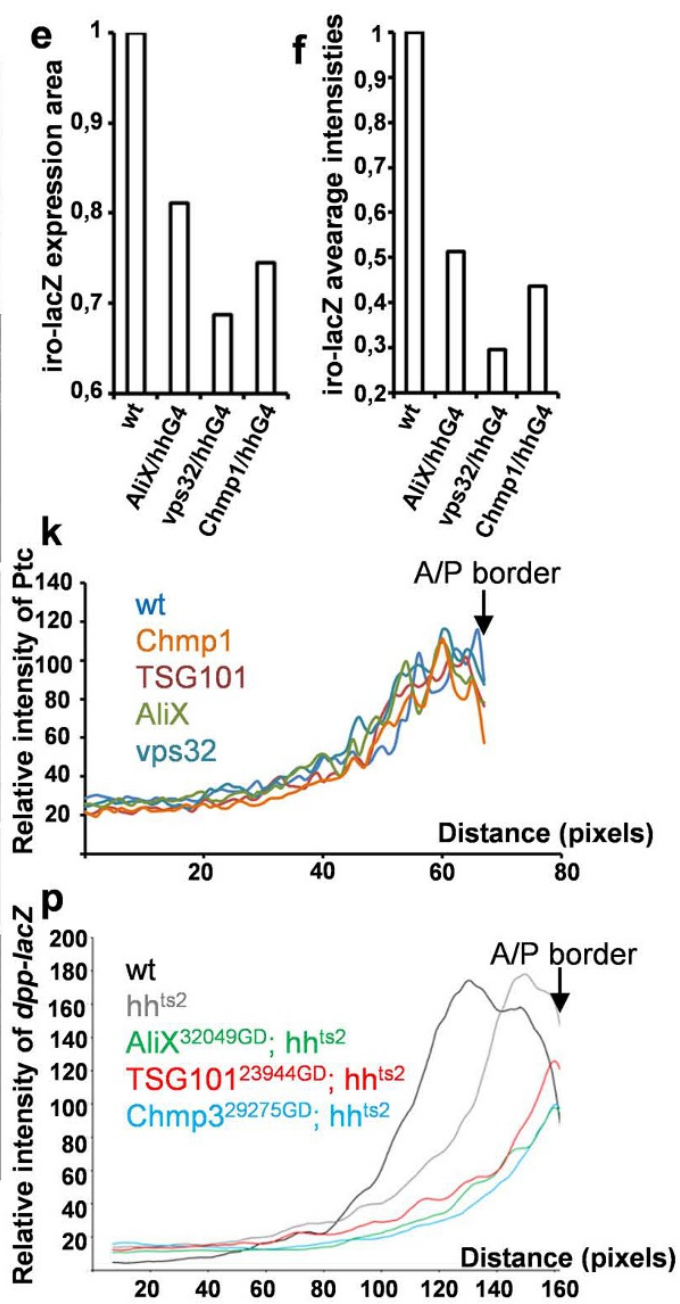

onwards. At restrictive temperature, the $h h^{t s 2}$ allele is semi-lethal, with up to $60 \%$ of the flies dying as pharate adults (Methods). The surviving flies displayed a 50\% decrease in the v3-v4 intervein space (Extended Data Fig. 1e, l). $d p p$-lacZ expression, which was mildly affected in the $h h^{t s 2} \mathrm{WID}(\mathbf{l}, \mathbf{p})$, became strongly reduced in all ESCRT RNAi discs in the $h h^{\text {ts } 2}$ background analysed $(\mathbf{m}-\mathbf{p})$. A magnification of the $d p p$-lac $Z$ stripe is shown for each genotype below the panels. p, Quantification of $d p p$-lacZ expression in wild-type, $h h^{t s 2}$ and ESCRT RNA $i ; h h^{t s 2}$ discs. q-t, Ptc expression in conditions of ESCRT RNAi; $h h^{t s 2}$ discs. ESCRT RNAis had no effect on the decrease of Ptc expression observed in $h h^{t s 2}$ WIDs, suggesting that Hh long-range activity is sensitive to ESCRT function, but not short-range Hh signalling $(\mathbf{q}-\mathbf{t})$. On all fluorescence images, dorsal is shown to the top and anterior is to the left. Scale bar, $50 \mu \mathrm{m}$. b-d, l-o, Numbers on bottom right indicate number of discs displaying reduced iro or $d p p$ activity per whole population analysed. 

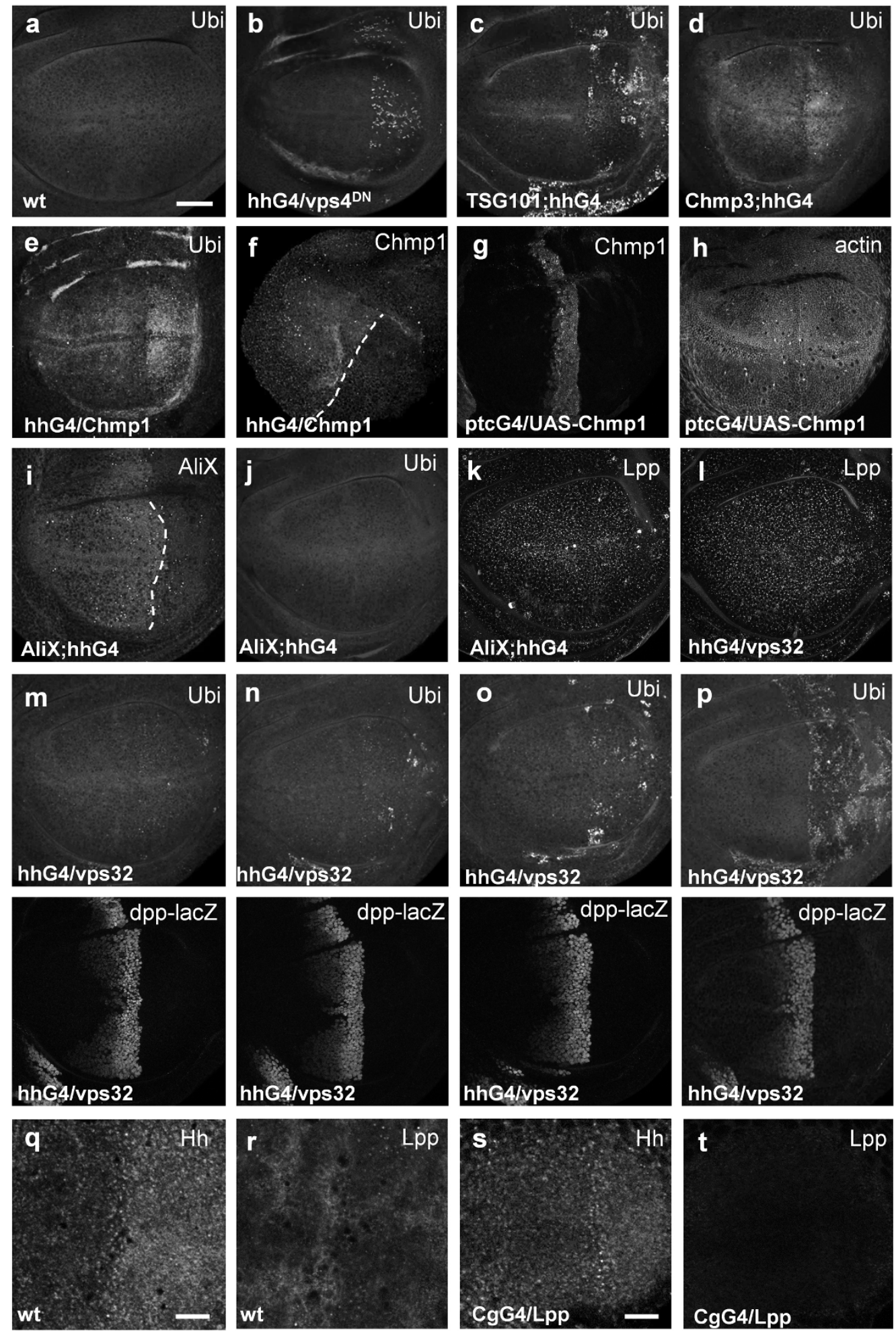

Extended Data Figure $5 \mid$ Specificity controls for ESCRT RNAi.

$\mathbf{a}-\mathbf{e}$, Expression of Vps4 ${ }^{\text {DN }}(\mathbf{b})$ or ESCRT RNAi (c-e, and see also in $\left.\mathbf{n}-\mathbf{p}\right)$ in the posterior compartment results in the accumulation of the poly-Ubi epitope compared with wild type (a). $\mathbf{f}-\mathbf{h}, \mathrm{Chmp} 1 \mathrm{RNAi}$ in the Hh-producing cells leads to the depletion of Chmpl epitopes (f), whereas overexpression of UAS-Chmp1 in the Ptc domain results in the accumulation of the Chmp1 signal (g, h). i, AliX RNAi in the Hh-producing cells leads to the depletion of AliX epitopes. $\mathbf{j}, \mathbf{k}$, AliX RNAi in Hh-producing cells does not result in the accumulation of either poly-Ubi or Lpp epitopes. This disc is the same as the one in Fig. 2b. 1-p, Panels show four examples of discs expressing RNAi against Vps32 in the posterior compartment stained for Lpp (I), poly-Ubi ( $\mathbf{m}-\mathbf{p}$, top row) and $d p p$-lac $Z$ ( $\mathbf{m}-\mathbf{p}$, bottom row). Note that $d p p$-lac $Z$ expression can

be reduced ( $\mathbf{m}$, bottom panel) even in a disc with no change in poly-Ubi ( $\mathbf{m}$, top panel) and Lpp distribution (l). Increased poly-Ubi accumulation is not correlated with a further decrease in $d p p$-lacZ expression. $\mathbf{l}, \mathbf{m}$, The disc shown is the same as the one in Fig. 2c. q-t, Expression of an Lpp RNAi construct in the fat body using the CgGal4 (CgG4) driver decreases Lpp protein levels in the WID $(\mathbf{r}, \mathbf{t})$, but does not cause Hh accumulation at the apical cell surface $(\mathbf{q}, \mathbf{s})$. Shown is a close-up of the central disc quadrant overlying the anterior-posterior compartment boundary. In all images dorsal is at the top and anterior is to the left. $\mathbf{s}, \mathbf{t}$, Note that because of general depletion of Lpp the size of the disc is considerably reduced. a, $\mathbf{q}, \mathbf{s}$, Scale bars, $30 \mu \mathrm{m}$. In all images dorsal is at the top and anterior is to the left. 

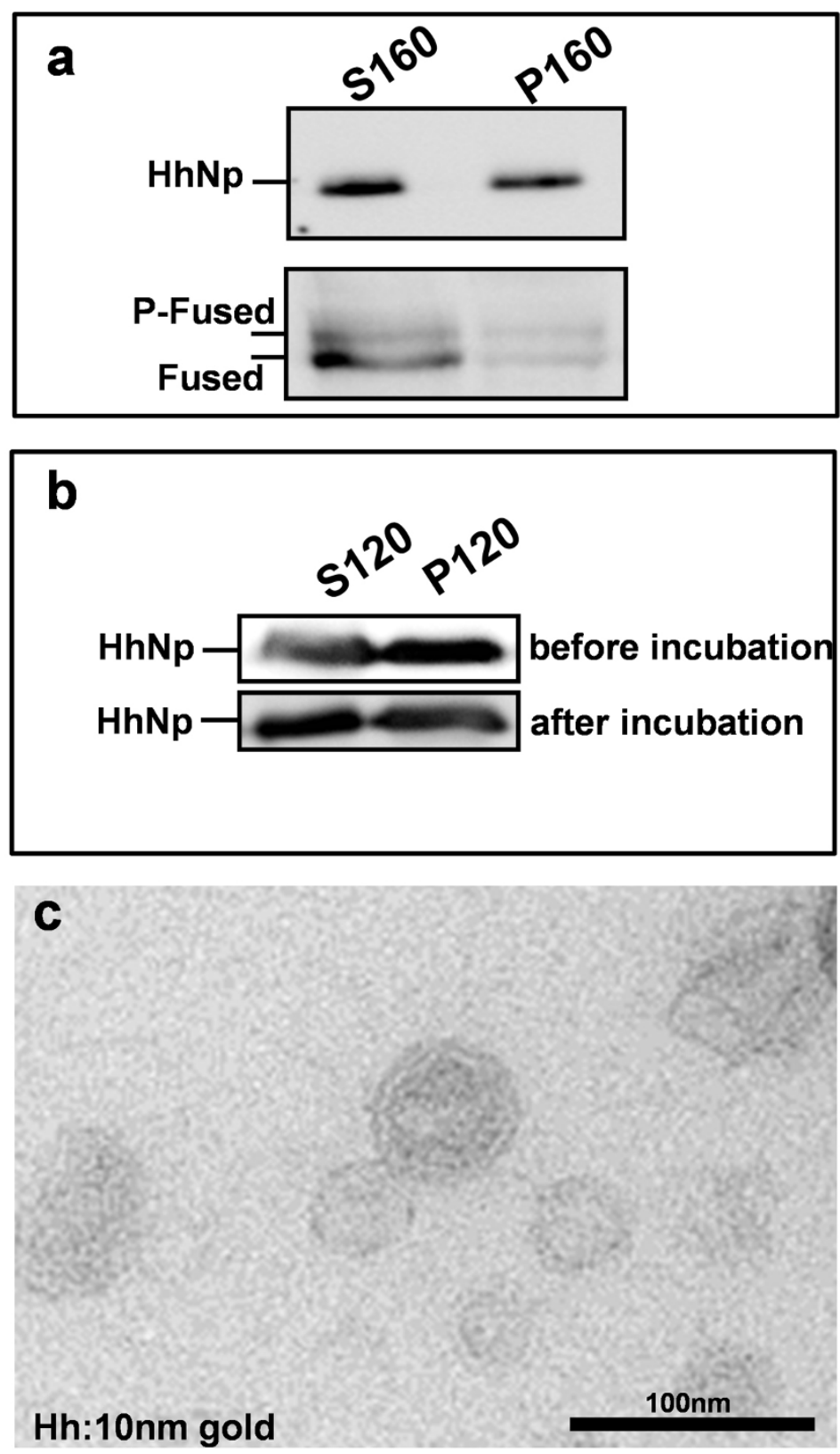

Extended Data Figure 6 Control experiments for exovesicle characterization. a, The S10 fraction was run at $160,000 \mathrm{~g}$ for $24 \mathrm{~h}$. Hh protein split equally between the $160,000 \mathrm{~g}$ supernatant and the $160,000 \mathrm{~g}$ pellet. These two fractions induced Fu phosphorylation (P-Fused) with the same intensity. $\mathbf{b}$, The relative amount of S120-Hh and P120-Hh before and after incubation of cells in Fig. 3c. c, Immunogold labelling of P120 fraction isolated from non-Hh-expressing $\mathrm{Cl} 8$ cells. In this experimental condition the $\mathrm{Hh}$ epitope is not detectable on the surface of exovesicles. This is a control for Fig. 3e. 

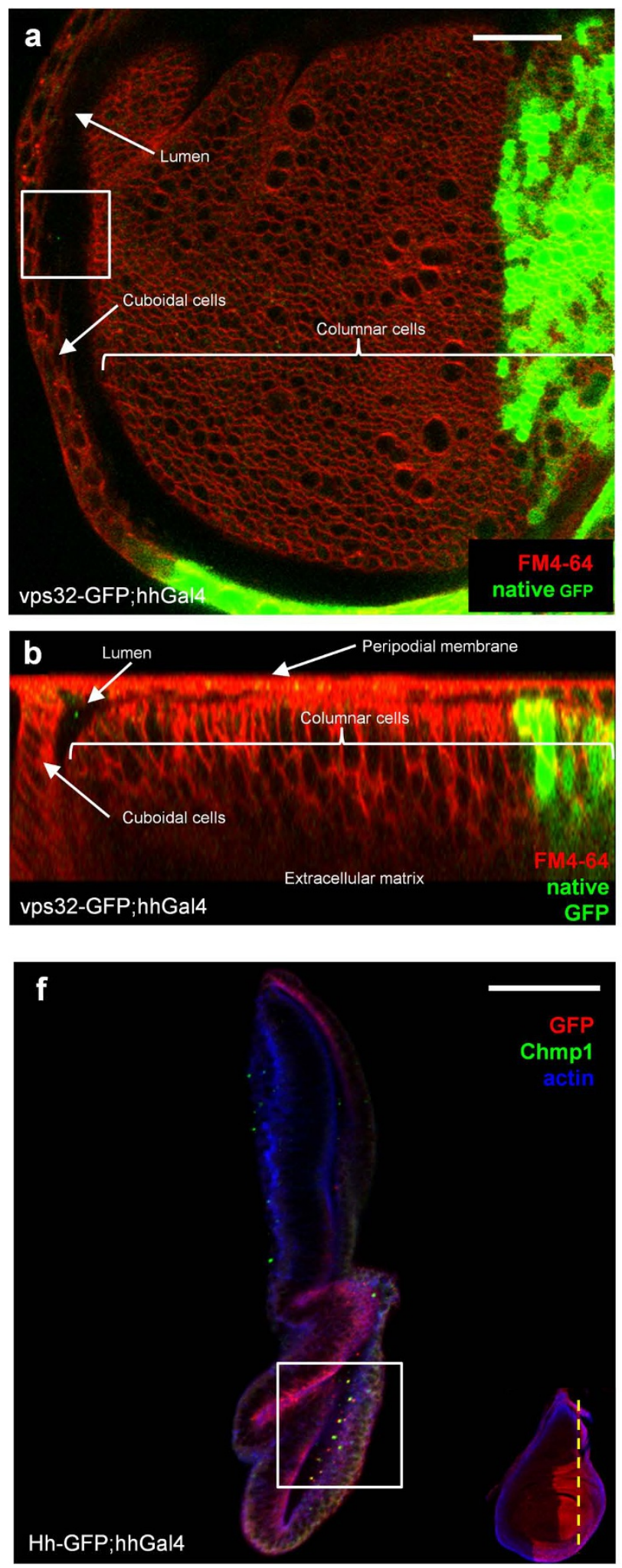

Extended Data Figure $7 \mid$ Visualization of ESCRT-positive exovesicles in the luminal space of Drosophila WIDs. a, b, Live imaging of Vps32-GFP in a WID. Cell membranes are stained with FM4-64 (red). Scale bar, $20 \mu \mathrm{m}$. a, $X-Y$ section through the WID pouch showing the posterior Vps32-GFP-producing cells on the right (strong green signal) and the anterior luminal space on the left. The white square indicates the region shown at a higher magnification in Fig. 4 b. b, $X-Z$ section showing the Vps32-GFP-expressing columnar cells on the right (bright green) and a Vps32-GFP-positive vesicle in the anterior lumen. $\mathbf{c}-\mathbf{f}$, Colocalization of $\mathrm{Hh}-\mathrm{GFP}$ and endogenous $\mathrm{Chmp} 1$ protein in the WID lumen. c-e, Transverse section through the ventral part of the wing pouch
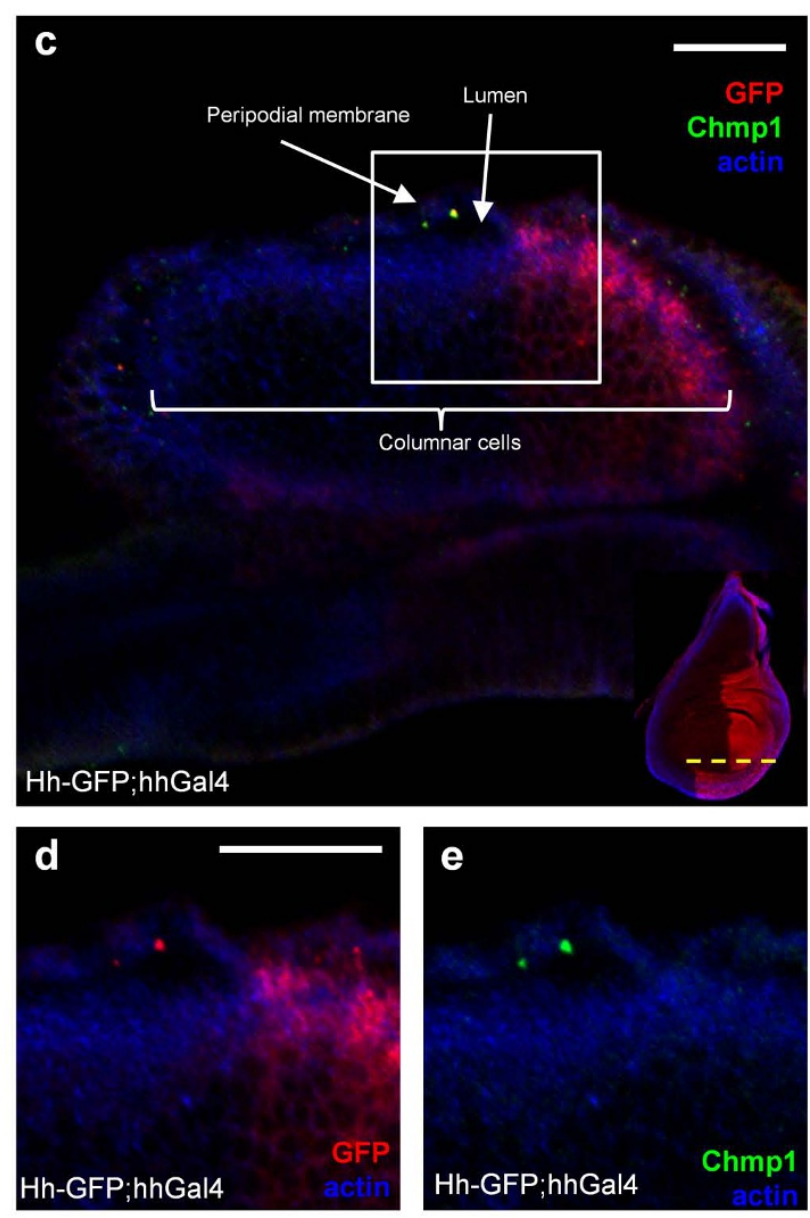

parallel to the dorsal-ventral axis. Scale bars, $20 \mu \mathrm{m}$. d, e, Higher magnification views of the region indicated by the white box in $\mathbf{c}$. Note the presence of Hh- and Chmp1-positive exovesicles in the luminal space in c-f. Cell outlines are visualized by the staining of cortical filamentous actin (phalloidin, blue). f, Transverse section through the WID across the dorsal-ventral axis. The white square indicates the region shown at a higher magnification in Fig. $4 \mathrm{f}-\mathrm{h}$. Scale bar, $50 \mu \mathrm{m}$. Schematic representations of the positions of the optical sections (dashed yellow lines) used in $\mathbf{c}$ and $\mathbf{f}$ are shown in the bottom-right corner of the corresponding images. 

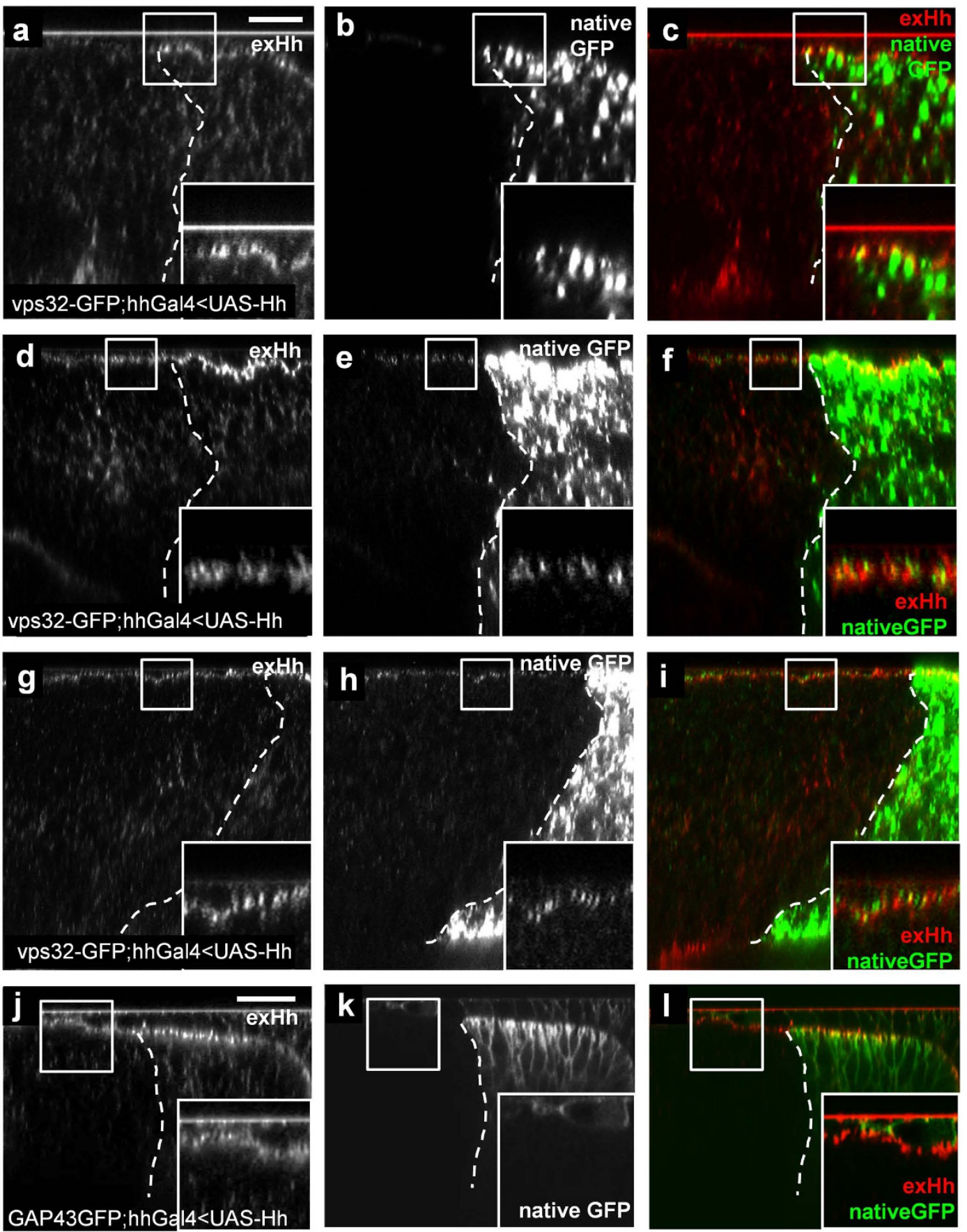

Extended Data Figure $8 \mid$ Controls and additional examples for the extracellular colocalization of $\mathrm{Hh}$ and Vps32-GFP. a-i, Partial colocalization of extracellular $\mathrm{Hh}$ and Vps32-GFP on the apical side of both the producing $(\mathbf{a}-\mathbf{c})$ and recipient cells $(\mathbf{d}-\mathbf{i})$. Scale bar, $10 \mu \mathrm{m}$. Pearson coefficients: $0.426(\mathbf{d}-\mathbf{f})$ and $0.542(\mathbf{g}-\mathbf{i})$. M1M2 coefficients: 0.156 for Hh and 0.146 for Vps32-GFP (d-f); 0.284 for Hh and 0.382 for Vps32-GFP (g-i). $\mathbf{j}-\mathbf{l}$, GAP43GFP, an inner plasma membrane marker, was expressed in the posterior compartment. No colocalization with $\mathrm{Hh}$ was observed in the anterior compartment (Pearson coefficient: 0.009; M1M2 coefficients: 0.001for Hh and 0.142 for GAP43-GFP). Note that GAP43-GFP-labelled peripodial membrane above the columnar cells. Scale bar, $20 \mu \mathrm{m}$. Apical is towards the top and anterior is to the left. The anterior-posterior border is marked with a dashed white line. Magnification of $\mathrm{Hh}$ punctae is shown in the bottom-right corner. 

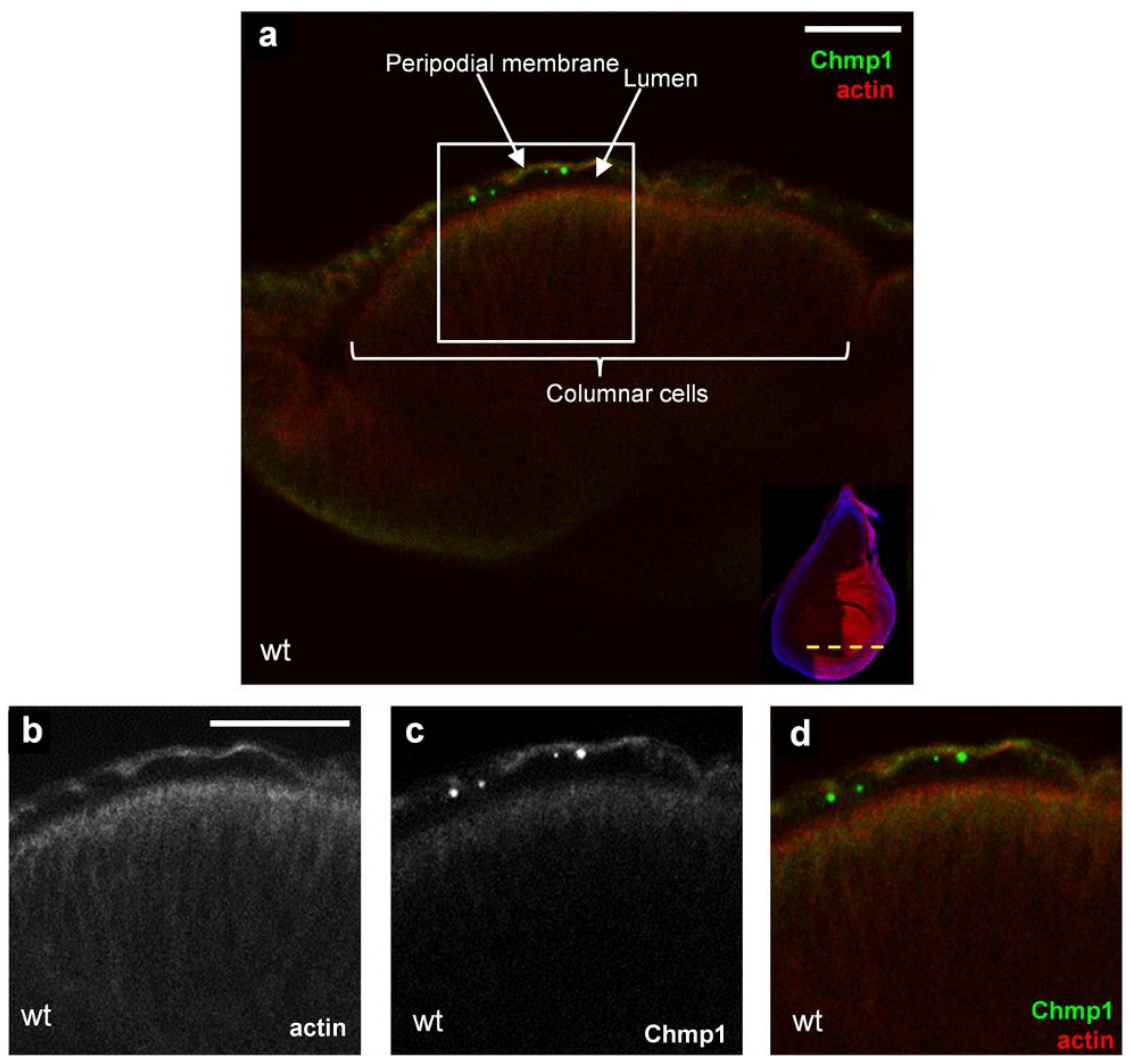

Extended Data Figure $9 \mid$ Endogeneous Chmp1 is secreted into the luminal space of WID. a-d, Transverse section through the ventral part of the wing pouch across the anterior-posterior axis. Scale bars, $20 \mu \mathrm{m}$. b-d, Higher presence of Chmp1-positive exovesicles in the luminal space. Cell outlines are visualized by the staining of cortical filamentous actin (phalloidin, red). magnification views of the region indicated by the white box in $\mathbf{a}$. Note the Schematic representation of the position of the optical section (dashed yellow line) used in $\mathbf{a}-\mathbf{d}$ is shown in the bottom-right corner of $\mathbf{a}$. 

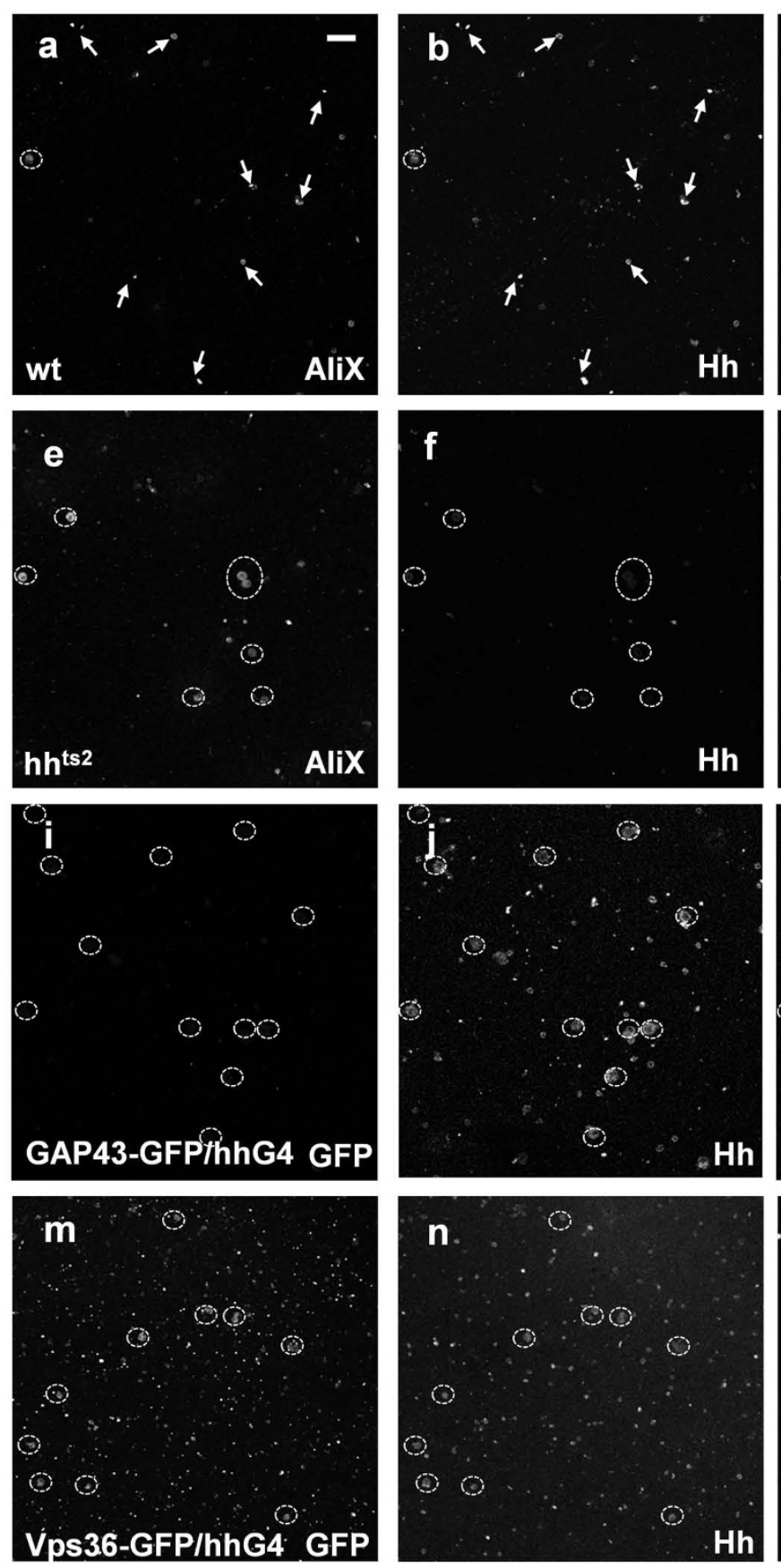

Extended Data Figure $10 \mid$ Specificity controls for Drosophila haemolymph staining. a-d, Endogenous Hh colocalizes with AliX on non-cellular (DAPI-negative) particles in the Drosophila haemolymph. White arrows indicate examples of colocalization. $\mathbf{e}-\mathbf{h}$, Most of the Hh epitope is depleted from the circulating particles in the $h h^{\text {ts } 2}$ homozygous mutant, which was kept
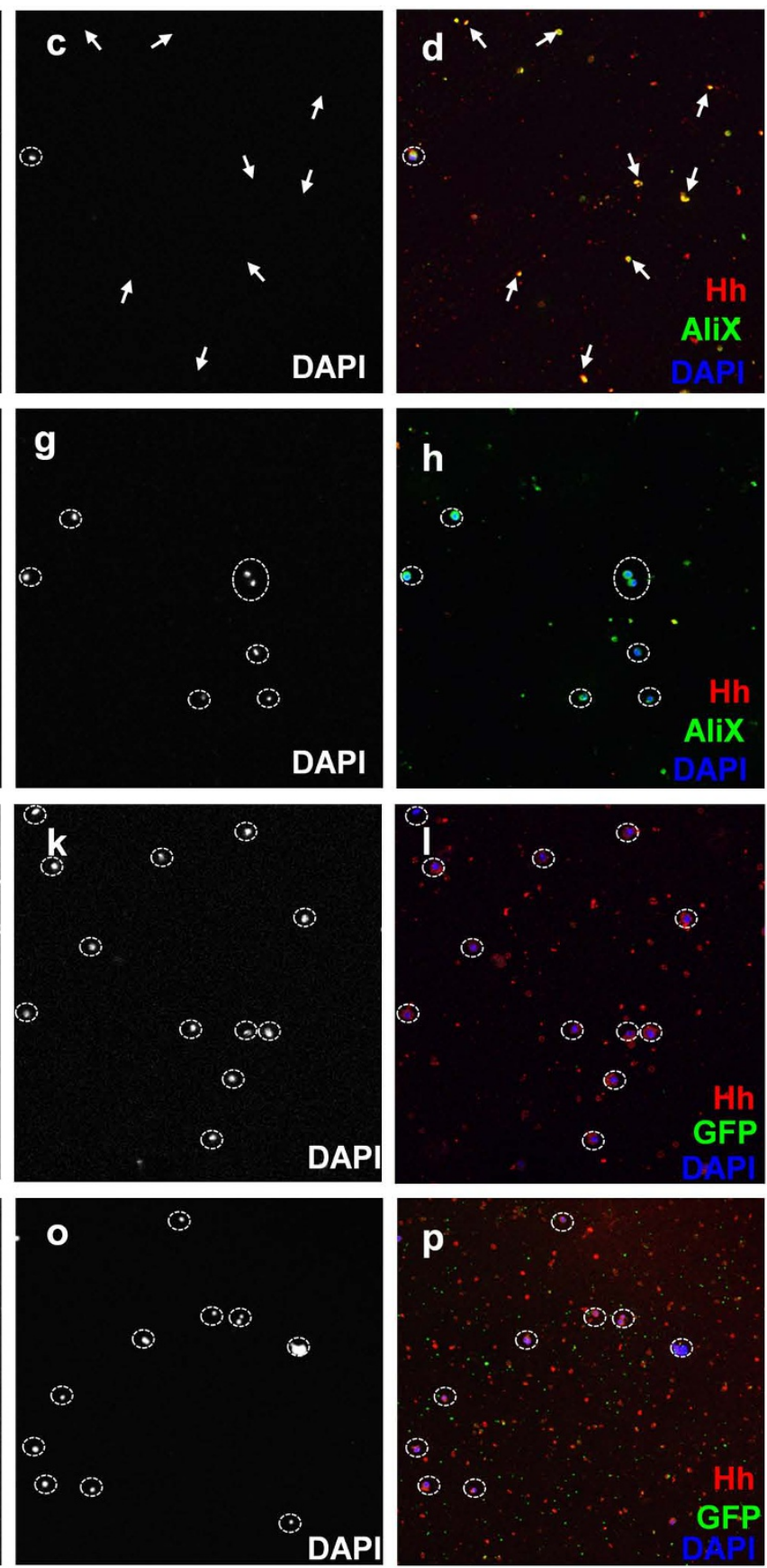

at a restrictive temperature for 4 days. i-1, GAP43-GFP expressed in all posterior cells is not secreted into the Drosophila circulation. $\mathbf{m}-\mathbf{p}$, The ESCRT II member Vps36-GFP expressed in all posterior cells does not colocalize with endogeneous $\mathrm{Hh}$ in the fly blood, but is secreted as different particles instead. Scale bar, $20 \mu \mathrm{m}$. White circles mark DAPI-positive blood cells. 